TRANSACTIONS OF THE

AMERICAN MATHEMATICAL SOCIETY

Volume 364, Number 11, November 2012, Pages 5735-5763

S 0002-9947(2012)05611-X

Article electronically published on June 20, 2012

\title{
CONTRACTING CONVEX IMMERSED CLOSED PLANE CURVES WITH SLOW SPEED OF CURVATURE
}

\author{
YU-CHU LIN, CHI-CHEUNG POON, AND DONG-HO TSAI
}

\begin{abstract}
The authors study the contraction of a convex immersed plane curve with speed $\frac{1}{\alpha} k^{\alpha}$, where $\alpha \in(0,1]$ is a constant, and show that, if the blow-up rate of the curvature is of type one, it will converge to a homothetic self-similar solution. They also discuss a special symmetric case of type two blow-up and show that it converges to a translational self-similar solution. In the case of curve shortening flow (i.e., when $\alpha=1$ ), this translational self-similar solution is the familiar "Grim Reaper" (a terminology due to M. Grayson).
\end{abstract}

\section{INTRODUCTION}

Let $\gamma_{0}$ be a convex immersed smooth closed plane curve with rotation index (the number of times its tangent vector winds around as one goes along the curve) $m \in \mathbb{N}$, parametrized by a smooth immersion $X_{0}(\varphi): S^{1} \rightarrow \mathbb{R}^{2}$. Here "convex" means that $\gamma_{0}$ has no inflection points (i.e., curvature is positive everywhere). In general, such a curve $\gamma_{0}$ can have self-intersections (if $m \geq 2$ ).

A family of convex immersed closed curves $X(\varphi, t): S^{1} \times[0, T) \rightarrow \mathbb{R}^{2}$ (with rotation index $m$ and initial data $\gamma_{0}$ ) is said to evolve (contract) under the $k^{\alpha}$ flow, where $\alpha>0$ is a constant, if we have

$$
(\star) \quad \begin{cases}\frac{\partial X}{\partial t}(\varphi, t)=\frac{1}{\alpha} k^{\alpha}(\varphi, t) \mathbf{N}(\varphi, t), & \forall(\varphi, t) \in S^{1} \times[0, T), \\ X(\varphi, 0)=X_{0}(\varphi) \in C^{\infty}\left(S^{1}\right), & \varphi \in S^{1},\end{cases}
$$

where $k(\varphi, t)$ is the curvature of the curve $\gamma_{t}:=X(\cdot, t)$ at $\varphi$ and $\mathbf{N}(\varphi, t)$ is the unit normal vector of the curve $\gamma_{t}$. Throughout this paper the constant $\alpha$ is assumed to be $0<\alpha \leq 1$ (in such a case, we shall call $(\star)$ a slow speed contraction). Here we use the convention that for convex plane curves the curvature $k>0$ is positive everywhere, and as for the direction of the normal $\mathbf{N}$, we choose $\mathbf{N}=(0,1)$ at a point with minimum $y$-coordinate and extend it continuously to the whole curve.

When $\alpha=1$ (i.e., the well-known curve-shortening flow), our setting is exactly the same as in the interesting paper by Angenent [ANG, where the flow $(\star)$ contracts $\gamma_{0}$ with singularity forming in finite time.

Our aim is to study the asymptotic behavior of the contracting flow $(\star)$ with $m \geq 2$ (the immersed case), trying to generalize results in [ANG] to the case $\alpha \in(0,1]$. The behavior of the contracting flow $(\star)$ with fast speed, i.e., when

Received by the editors October 12, 2010.

2010 Mathematics Subject Classification. Primary 53C44, 35K15, 35K55.

The third author's research was supported by the NCTS and the NSC of Taiwan under grant number 96-2115-M-007-010-MY3. 
$1<\alpha<\infty$, has been discussed in $[\mathrm{PT}]$. Note that $\alpha \in(1, \infty)$ corresponds to $p \in(1,2)$ in the equation ( $)$ below.

When $m=1$ (the embedded case), the initial curve $X_{0}$ is embedded and the convergence behavior of the flow $(\star)$ for general $\alpha>0$ is well understood due to a series of nice papers by Ben Andrews [AN1, AN3 and AN4]. For the information of the readers, we give a brief summary provided by Andrews 1$]$

Theorem 1 (Ben Andrews AN1, AN3, AN4]). For $m=1$ and any $\alpha>0$, the curve $\gamma_{t}$ contracts to a point in finite time. If $0<\alpha<1 / 3$, then for generic initial data there is no limit of the curves rescaled about the final point (the isoperimetric ratio approaches infinity), and the exceptional ones where the isoperimetric ratio remains bounded converge to homothetic solutions, which have been classified. For $\alpha>1 / 3$, the rescaled solutions converge to circles, and for $\alpha=1 / 3$, they converge to ellipses.

Remark 2. As a consequence of Theorem 1, we have the following interesting elliptic result. For $0<\lambda<3$ (here $\lambda=1 / \alpha$ ), the only positive $2 \pi$-periodic solution to the equation

$$
w^{\lambda}(x)\left[w_{x x}(x)+w(x)\right]=1, \quad x \in S^{1},
$$

is $w(x) \equiv 1$. But for $\lambda \geq 3$, we begin to have nonconstant $2 \pi$-periodic solutions. For example, when $\lambda=3$, there is a family of positive $2 \pi$-periodic solutions to equation (11) of the form $(b \in \mathbb{R}$ is a parameter $)$

$$
w(x)=\left(\frac{1}{1+b^{2}}\right)^{1 / 4} \sqrt{1+b^{2} \cos ^{2} x}, \quad x \in[0,2 \pi],
$$

where we obtain (2) by computing the curvature (or support function) of an ellipse. The function $w(x)$ of (2) has maximum value occurring at $x=0$, with value $\left(1+b^{2}\right)^{1 / 4} \geq 1$.

Let $x \in S_{m}^{1}:=\mathbb{R} / 2 m \pi \mathbb{Z}$ be the tangent angle of $\gamma_{t}$ (a function defined on $S_{m}^{1}$ means that it is periodic with period $2 m \pi)$. In terms of the variable $(x, t)$, it is known that the curvature quantity $v(x, t)=k^{\alpha}(x, t)$ of $\gamma_{t}$ in $(\star)$ will satisfy the quasilinear parabolic PDE

(\$)

$$
\left\{\begin{array}{l}
\frac{\partial v}{\partial t}=v^{p}\left(v_{x x}+v\right), \quad p=1+\frac{1}{\alpha} \in[2, \infty), \quad 0<\alpha \leq 1, \\
v(x, 0)=v_{0}(x)>0 \quad \text { for all } x \in S_{m}^{1}, \\
v(x, t)=v(x+2 m \pi, t) \quad \text { for all } \quad x \in \mathbb{R}, \quad t>0
\end{array}\right.
$$

where $k_{0}(x)$ is the curvature of $\gamma_{0}$ and $v_{0}(x)=k_{0}^{\alpha}(x), x \in S_{m}^{1}$. Moreover, it is also known that $(\boldsymbol{\beta})$ is equivalent to $(\boldsymbol{\star})$ (under the assumption that $v_{0}(x)>0$ satisfies the integral condition (3) below). As $\gamma_{0}$ is a closed curve, in ( $\star$ ) the initial data $v_{0}(x)=k_{0}^{\alpha}(x)>0$ in (\$) must satisfy the integral condition

$$
\int_{S_{m}^{1}} v_{0}^{1-p}(x) e^{i x} d x=0, \quad e^{i x}=\cos x+i \sin x,
$$

where $\int_{S_{m}^{1}}$ means $\int_{-m \pi}^{m \pi}$. Also note that (3) is preserved under (\$), i.e., if initially $v_{0}(x)$ satisfies (3) , so does $v(x, t)$.

\footnotetext{
${ }^{1}$ We thank Ben Andrews for giving us this summary.
} 
From now on we shall focus on (⿳) with $p \in[2, \infty)$ and $m \geq 2$ with the smooth initial data $v_{0}(x)>0$ in (\$) satisfying (3). In Lemma 21 we shall discuss a result when the initial function $v_{0}(x)$ does not satisfy the integral condition (3). The overall understanding is that when (3) is satisfied, then we are talking about the geometric flow $(\star)$. If not, then one can simply view (\$) as a pure analytical problem.

Since equation (\$) is parabolic, regularity theory implies the existence of a unique smooth periodic solution $v(x, t)$ on $S_{m}^{1} \times[0, T)$ for some $T>0$. Each $v(\cdot, t)$, $t \in[0, T)$, remains smooth, positive, and periodic over $\mathbb{R}$ with period $2 m \pi$. By the equivalence, the flow $(\star)$ also has short time existence of a smooth solution. Each $\gamma_{t}$ remains convex, closed, and immersed with rotation index $m$ for all $t \in[0, T)$.

The classical curve-shortening flow occurs when $\alpha=1$ (or $p=2$ ); see GageHamilton [GH] for the embedded case (i.e., $m=1$ ) and Angenent [ANG], AngenentVelázquez $[\mathrm{AV}]$ for the immersed case (i.e., $m \geq 2$ ). When $m=1$, the value $\alpha=1 / 3$ in Theorem 1 corresponds to $p=4$ in (\$). For more information on the evolution (expansion or contraction) of convex closed curves in $\mathbb{R}^{2}$, see Andrews [AN1], ChouZhu $\mathrm{CZ}$, and the references therein.

Remark 3. If in ( $\mathbf{s})$ the constant $\alpha$ is negative (let $\alpha=-\beta, \beta>0$ ), then the corresponding flow in $(\star)$ is to expand $\gamma_{0}$ along its outward normal vector direction with speed $1 /\left(\beta k^{\beta}\right)$. The evolution of $v=1 / k^{\beta}$ is given by

$$
\frac{\partial v}{\partial t}=v^{p}\left(v_{x x}+v\right), \quad v(x, t)=v(x+2 m \pi, t), \quad p=1+\frac{1}{\alpha} \in(-\infty, 1) .
$$

Finally, if one expands $\gamma_{0}$ along its outward normal vector direction with the exponential speed $\exp (1 / k)$, the evolution of $v=e^{1 / k}$ is

$$
\frac{\partial v}{\partial t}=v\left(v_{x x}+v\right), \quad v(x, t)=v(x+2 m \pi, t),
$$

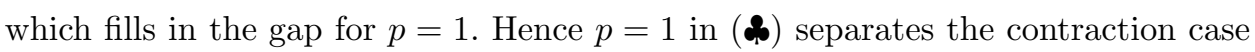
from the expansion case.

When $m \geq 2$, the behavior of solutions $v(x, t)$ to the equation

$$
\frac{\partial v}{\partial t}=v^{p}\left(v_{x x}+v\right), \quad v(x, 0)=v_{0}(x)>0 \in C^{\infty}\left(S_{m}^{1}\right), \quad p \in(-\infty, \infty),
$$

for $p \in(-\infty, 0], p \in(0,1), p=1, p \in(1,2), p \in[2, \infty)$ are all quite different. This means that equation (6) has at least the following "critical values". Each case has its own feature, explained below:

- $p=0$. The case of the linear heat equation for the function $e^{-t} v$ or the case of expanding flow with speed $1 / k$. It also separates the sublinear case $(p<0)$ and the superlinear case $(p>0)$.

- $p=1$. The case which separates the contraction case from the expansion case. In such a case, (3) becomes

$$
\int_{S_{m}^{1}} \log v_{0}(x) e^{i x} d x=0 .
$$

Since the behavior of $\log x$ is different from $x^{1-p}$ for $p \neq 1$, this case is quite special. 
- $p=2$. The case of the classical curve-shortening flow. It is the gradient flow of the length functional. As we shall see below, for $p \geq 2$, (6) begins to have a type-two blow-up (or type-two singularity in $(\star)$ ). Thus $p=2$ separates the type-one blow-up and the type-two blow-up (see the definition for type-one and type-two blow-ups below).

Remark 4. By Andrews's Theorem 1 one can also view $p=4$ as a critical value although it is for $m=1$. Also see the discussions before Remark 17.

The behavior of solutions of (6) for $p \geq 2$ is mostly unknown to us, especially the blow-up rate of a type-two singularity. The case $p=1$ is also complicated. Some proofs valid for $p \neq 1$ cannot be carried over to the case $p=1$. To see their differences, we refer to the papers by Angenent ANG $(p=2)$, Angenent-Velázquez AV] $(p=2)$, [PT] $(1<p<2)$, T3] $(p=1)$, [LPT] $(0<p<1)$ and Urbas [U1] $(p \leq 0)$ for details.

Let $v_{\min }(t)=\min _{x \in S_{m}^{1}} v(x, t)$, and similarly for $v_{\max }(t)$. For all $p \in(-\infty, \infty)$ in equation (6), as long as a solution exists, $v_{\min }(t)$ is always increasing due to the maximum principle. By the parabolic regularity theory, it is also known that the smooth solution $v(x, t)$ to equation (6) exists on some maximal time interval $\left[0, T_{\max }\right)$, where $v_{\max }(t)$ blows up at $T_{\max }\left(v_{\max }(t)\right.$ will be eventually increasing for $t$ close to $\left.T_{\max }\right)$. For $p \leq 0, T_{\max }=\infty$ and for $p>0, T_{\max }<\infty$.

When $T_{\max }<\infty$ (i.e., when $p>0$ ), if we let $R(t)$ be the unique solution to the ODE

$$
\frac{d R}{d t}=R^{p+1}(t), \quad R\left(T_{\max }\right)=\infty,
$$

then $R(t)=\left[p\left(T_{\max }-t\right)\right]^{-1 / p}$, and the comparison principle implies that

$$
0<v_{\min }(t) \leq R(t) \leq v_{\max }(t) \quad \text { for all } t \in\left[0, T_{\max }\right) .
$$

We define the following terminology: if there exists a constant $C$, independent of time, such that

$$
0<v_{\max }(t) \leq C R(t) \quad \text { for all } t \in\left[0, T_{\max }\right),
$$

then we say $v(x, t)$ has type-one blow-up. If not, i.e., if $v_{\max }(t) / R(t)$ is not bounded on $t \in\left[0, T_{\max }\right)$, then we say $v(x, t)$ has type-two blow-up. A type-two blow-up is clearly much more complicated.

It has been shown on p. 158 of [LPT] that for $p \in(0,2)$ there is no type-two blowup for any $m \in \mathbb{N}$ (include $m=1$ ) and any positive initial data $v_{0}(x) \in C^{\infty}\left(S_{m}^{1}\right)$ (whether it satisfies (3) or not), i.e., all blow-ups are of type-one in (\$) for $p \in(0,2)$ and for any $v_{0}(x)>0$. However, for $p \in(0,2)$, the limit function $w(x)(w(x)$ is the limit of the rescaled solution $v(x, t) / R(t)$ ) may be either $w(x)>0$ everywhere on $S_{m}^{1}$ (call it nondegenerate) or $w(x) \geq 0$, but with $w(x)=0$ somewhere on $S_{m}^{1}$ (call it degenerate). If $w(x)$ is nondegenerate, it gives rise to a selfsimilar (homothetic) solution. If $w(x)$ is degenerate, its behavior (regularity) for $p \in(0,1), p=1$, and $p \in(1,2)$ are all different.

In Angenent [ANG] (the case $p=2$ ), he employed an elegant unstable manifold analysis of a shrinking spiral (travelling wave solution) and used it to prove a Harnack-type estimate, i.e., Lemma 7.1 of ANG. This is the key estimate to ensure convergence to a positive self-similar solution under type-one blow-up (see p. 605, Theorem A of his paper). 
We shall see that his proof can be carried to the case $p \in[2, \infty)$, assuming that we have type-one blow-up. Hence we can obtain convergence of equation (\$) (or the flow $(\star)$ ) to a self-similar (homothetic) solution $w(x)$ under type-one blow-up. These homothetic solutions have been classified in Andrews AN3. Unlike the case for $p \in(0,2)$, now $w(x)$ is positive everywhere on $S_{m}^{1}$ (for $p \in[2, \infty)$ ) and is an entire periodic solution to the corresponding steady state ODE. See Theorem 18 in Section 3 .

In summary, the above says that for $p \in[2, \infty)$ there is either type-one blowup or type-two blow-up. Moreover, if we have type-one blow-up, then the limit function $w(x)$ is always nondegenerate, i.e., $w(x)>0$ everywhere.

As for Theorem B of [ANG], it is rather straightforward to generalize it to the case $p \geq 2$. See Theorem 8 .

Finally, we also discuss a special symmetric case of type-two blow-up and obtain a convergence to the cosine function (see Theorem 25] and Theorem 301). In flow $(\star)$, this convergence gives rise to a translational self-similar solution. When $\alpha=1$ (curve-shortening flow), this translational self-similar solution is Grayson's "Grim Reaper", i.e., the graph of $y=-\log \cos x, x \in(-\pi / 2, \pi / 2)$. One can view Theorem 25 and Theorem 30 as partial generalizations of Theorem $\mathrm{C}$ of [ANG] to the case $p \geq 2$.

In conclusion, we can generalize Theorem A, Theorem B, and part of Theorem $\mathrm{C}$ on p. 605 of Angenent [ANG] to the case $p \geq 2$.

To end this introductory section, we point out that solutions of (6) for the sublinear case $p<0$ are well-behaved, as it is bounded by the following super-sub solutions:

$$
\left[\left(\min _{x \in S_{m}^{1}} v_{0}(x)\right)^{-p}-p t\right]^{-1 / p} \leq v(x, t) \leq\left[\left(\max _{x \in S_{m}^{1}} v_{0}(x)\right)^{-p}-p t\right]^{-1 / p}
$$

for all $t \in[0, \infty)$. In particular, we have

$$
1 \leq \frac{v_{\max }(t)}{v_{\min }(t)} \leq U(t):=\frac{\left[\left(\max _{x \in S_{m}^{1}} v_{0}(x)\right)^{-p}-p t\right]^{-1 / p}}{\left[\left(\min _{x \in S_{m}^{1}} v_{0}(x)\right)^{-p}-p t\right]^{-1 / p}}, \quad t \in[0, \infty),
$$

where $U(t)$ is a decreasing function on $[0, \infty)$ with $\lim _{t \rightarrow \infty} U(t)=1$. In fact, the quantity $v_{\max }(t) / v_{\min }(t)$ also decreases to 1 as $t \rightarrow \infty$. As a consequence, by regularity theory, the rescaled solution $u(x, t):=v(x, t) / R(t)$ will converge as $t \rightarrow \infty$ to the constant function 1 in $C^{k}\left(S_{m}^{1}\right)$ for any $k \in \mathbb{N}$. Here $R(t)$ can be any solution to the ODE $d R / d t=R^{1+p}(p<0)$ with $R(0)>0$. The geometric meaning is that when $\alpha \in(-1,0)$, the expanding flow $(\star)$ converges (after rescaling) to the $m$-fold cover of $S^{1}$ in any $C^{k}$-norm. See Urbas U1] as well.

\section{Some BAsic EStimates}

From now on we assume $p \geq 2$ and $m \geq 2$, with the smooth initial data $v_{0}(x)>0$ in (\$) satisfying (3). For convenience, denote the maximal space-time domain $S_{m}^{1} \times\left[0, T_{\max }\right)$ by $\Omega_{m}$. Below, if the proof of a lemma is omitted, then it is either straightforward or similar to those established in [GH] or [ANG] for the case $p=2$. Hence we will not repeat it. 
Lemma 5 (Gradient estimate in integral form). There exists a constant $C$ depending only on $v_{0}$ such that

$$
\int_{S_{m}^{1}} v_{x}^{2}(x, t) d x \leq \int_{S_{m}^{1}} v^{2}(x, t) d x+C
$$

for all $t \in\left[0, T_{\max }\right)$, where $v_{x}^{2}$ means $(\partial v / \partial x)^{2}$. In particular, for $\varepsilon>0$ sufficiently small, there exists a number $\delta>0$, depending only on $\varepsilon$, such that

$$
(1-\varepsilon) v_{\max }(t) \leq v(x, t)+\sqrt{2 m \pi C}
$$

for all $x \in\left(x_{t}-\delta^{2}, x_{t}+\delta^{2}\right)$ and all $t \in\left[0, T_{\max }\right)$, where $v\left(x_{t}, t\right)=v_{\max }(t)$.

Lemma 6 (Gradient estimate). For solution $v(x, t)$ to equation (\$) on $\Omega_{m}$ we have, at each point $(x, t)$, either

$$
\left(v_{x x}+v\right)(x, t)>0
$$

or

$$
v_{x}^{2}(x, t)+v^{2}(x, t) \leq \max _{x \in S_{m}^{1}}\left[\left(v_{0}\right)_{x}^{2}(x)+v_{0}^{2}(x)\right]:=\sigma .
$$

In particular, we have

$$
\left|v_{x}(x, t)\right| \leq \max \left\{\lambda, v_{\max }(t)\right\}
$$

for all $(x, t) \in \Omega_{m}$, where $\lambda>0$ is a constant depending only on $v_{0}$. As a consequence we also know that $v_{\max }(t)$ is eventually increasing for $t$ close to $T_{\max }$.

Lemma 7 (Behavior near maximum point). Let $v_{\max }(t)=v\left(x_{t}, t\right)$ for some $x_{t} \in$ $S_{m}^{1}$. If at any time $t \in\left[0, T_{\max }\right)$ we have $v_{\max }(t)>\sigma$, where $\sigma$ is from (15), then

$$
v(x, t)>v_{\max }(t) \cos \left(x-x_{t}\right)
$$

for all $x$ with $0<\left|x-x_{t}\right|<\arccos \left(\sigma / v_{\max }(t)\right)$.

With the help of the above basic estimates, we can generalize Theorem B of ANG] to the case $p \geq 2$ :

Theorem 8 (Rough upper bound of $v_{\max }(t)$ ). If $v_{\max }(t)$ blows up at time $T_{\max }$, then there holds the following:

$$
\lim _{t \rightarrow T_{\max }}\left(T_{\max }-t\right) v_{\max }(t)=0
$$

Remark 9. Now, by (9) and (18) we have the rough estimate

$$
\left[p\left(T_{\max }-t\right)\right]^{-1 / p} \leq v_{\max }(t) \leq \frac{C}{T_{\max }-t} \quad \text { for all } t \in\left[0, T_{\max }\right),
$$

where $p \geq 2$ and $C$ is some constant independent of time.

Proof. Let

$$
I(t)=\int_{S_{m}^{1}} v^{1-p}(x, t) d x>0, \quad p \geq 2 .
$$

By (13) in Lemma 5 we have for $t$ close to $T_{\max }$ the estimate

$$
\int_{S_{m}^{1}} v(x, t) d x \geq c v_{\max }(t)
$$


where $c>0$ is a constant independent of time. Hence there is a time $t_{*}$ close to $T_{\max }$ such that

$$
-I^{\prime}(t)=(p-1) \int_{S_{m}^{1}} v(x, t) d x \geq(p-1) c v_{\max }(t)>0 \quad \text { for all } \quad t \in\left[t_{*}, T_{\max }\right) .
$$

By integration of $i^{\prime}(t)$ on the interval $\left[t_{*}, T_{\max }\right)$, we obtain

$$
(p-1) c \int_{t_{*}}^{T_{\max }} v_{\max }(t) d t \leq I\left(t_{*}\right)-\left(\lim _{t \rightarrow T_{\max }} I(t)\right) \leq I\left(t_{*}\right)<\infty,
$$

and so the integral $\int_{0}^{T_{\max }} v_{\max }(t) d t$ is finite. Since by Lemma $6 v_{\max }(t)$ is eventually increasing, we may also assume that $v_{\max }(t)$ is increasing on $\left[t_{*}, T_{\max }\right)$ and conclude

$$
\left(T_{\max }-t\right) v_{\max }(t) \leq \int_{t}^{T_{\max }} v_{\max }(s) d s \text { for all } t \in\left[t_{*}, T_{\max }\right) .
$$

Letting $t \rightarrow T_{\max }$, the right hand side of (19) converges to zero and the proof is done.

\section{Type-one Blow-up implies $C^{\infty}$ CONVERgence}

Throughout this section we assume the solution $v(x, t)$ to equation (a) has typeone blow-up. We shall consider its asymptotic behavior by the obvious rescaling $u(x, t):=v(x, t) / R(t)$, where $R(t)$ is from (8) , and let $\tau \in[0, \infty)$ be the new time given by the relation $t=T_{\max }\left(1-e^{-p \tau}\right), t \in\left[0, T_{\max }\right)$, which is motivated by the requirement $d \tau / d t=R^{p}(t)$. Then the function

$$
u(x, \tau)=p^{1 / p} T_{\max }^{1 / p} e^{-\tau} v\left(x, T_{\max }\left(1-e^{-p \tau}\right)\right)>0, \quad x \in S_{m}^{1}, \quad \tau \in[0, \infty),
$$

will be a positive, bounded, solution of the rescaled equation

$$
\left\{\begin{array}{l}
\frac{\partial u}{\partial \tau}=u^{p}\left(u_{x x}+u-u^{1-p}\right), \quad p \geq 2 \\
u(x, \tau)=u(x+2 m \pi, \tau)
\end{array}\right.
$$

for all $(x, \tau) \in S_{m}^{1} \times[0, \infty)$, with $u(x, 0)=u_{0}(x):=p^{1 / p} T_{\max }^{1 / p} v_{0}(x)>0$. Moreover, we have

$$
0<u_{\min }(\tau) \leq 1 \leq u_{\max }(\tau)
$$

for all $\tau \in[0, \infty)$ due to (9). By (16) we also have the uniform gradient estimate

$$
\left|u_{x}(x, \tau)\right| \leq C \quad \text { for all } \quad(x, \tau) \in S_{m}^{1} \times[0, \infty),
$$

where $C$ is a constant depending only on $v_{0}$.

We shall generalize Angenent's Lemma 7.1 in $[\mathrm{ANG}$. to the following:

Theorem 10 (Gradient estimate for type-one blow-up). Let $v(x, t)$ be a type-one solution to equation ( with $p \geq 2$. Then the rescaled bounded positive function $u(x, \tau)$ of (20) satisfies

$$
\left|u_{x}(x, \tau)\right| \leq \lambda u(x, \tau) \quad \text { for all } \quad(x, \tau) \in S_{m}^{1} \times[0, \infty),
$$

where $\lambda$ is a constant depending only on $u_{0}$.

Remark 11. Theorem 10 fails for $p \in(0,2)$. 
Remark 12. By (24) we have the estimate

$$
1 \leq u_{\max }(\tau) \leq e^{2 \lambda m \pi} u_{\min }(\tau)
$$

for all $\tau \in[0, \infty)$. Hence $u_{\min }(\tau)$ has a positive lower bound for $\tau \in[0, \infty)$ and equation (21) is uniformly parabolic on $S_{m}^{1} \times[0, \infty)$.

Remark 13. From the proof we see that Theorem 10 remains valid even if the initial condition $v_{0}(x)$ does not satisfy the integral condition (3). This observation is important and will be used in Lemma 21 below.

3.1. Angenent's method of shrinking spirals. Theorem 10 has been proved by Angenent ANG for $p=2$. So we look at the case $p>2$. Our method of proof is similar to that originally used by Angenent in $\mathrm{ANG}$. (the energy functional for $p>2$ has a different form from $p=2$ ).

Consider a special solution (travelling wave solution) of the form $U(x, \tau)=$ $U(x-c \tau), c>0$ ( $c$ is a constant), to the equation

$$
\frac{\partial u}{\partial \tau}=u^{p}\left(u_{x x}+u-u^{1-p}\right)
$$

A positive function $U(\xi)$ over some interval $i$ will generate a solution if and only if

$$
U^{p}(\xi) U^{\prime \prime}(\xi)+U^{p+1}(\xi)-U(\xi)+c U^{\prime}(\xi)=0 \text { for all } \xi \in I .
$$

For such a $U(\xi)>0$ satisfying equation (27) on $i$ we have

$$
\frac{d}{d \xi}\left[\left(U^{\prime}(\xi)\right)^{2}+U^{2}(\xi)-\frac{2}{2-p} U^{2-p}(\xi)\right]=-2 c \frac{\left(U^{\prime}(\xi)\right)^{2}}{U^{p}(\xi)} \leq 0,
$$

and so the function $E(\xi)$ given by

$$
E(\xi):=\left(U^{\prime}(\xi)\right)^{2}+U^{2}(\xi)-\frac{2}{2-p} U^{2-p}(\xi), \quad \xi \in I, \quad p>2,
$$

is decreasing in $\xi \in I$ if $c \neq 0$. For $c \neq 0$ the only periodic solution for (27) is the constant $U(\xi) \equiv 1$.

For $c=0, E(\xi)$ is a positive constant independent of $\xi \in I$. It is obvious that any positive solution $U(\xi)$ satisfying the equation $E(\xi)=$ const. $>0$ cannot become too small over its domain since $-2(2-p)^{-1} U^{2-p}(\xi) \rightarrow \infty$ as $U(\xi) \rightarrow 0^{+}$. Thus any solution $U(\xi)$ to the $\mathrm{ODE} U^{p} U^{\prime \prime}+U^{p+1}-U=0$ is a positive periodic function on $\xi \in(-\infty, \infty)$ satisfying

$$
\left(U^{\prime}(\xi)\right)^{2}+U^{2}(\xi)-\frac{2}{2-p} U^{2-p}(\xi)=b^{2}-\frac{2}{2-p} b^{2-p}=a^{2}-\frac{2}{2-p} a^{2-p}, \quad \xi \in(-\infty, \infty),
$$

where $b \geq 1(a \leq 1)$ is the maximum (minimum) value of $U(\xi)$ over $(-\infty, \infty)$.

Similar to Theorem 6.1 of $\mathrm{ANG}$, we claim the following:

Theorem 14. Assume $p>2$. For any small $c>0$, there is a unique positive solution $U_{c} \in C^{\infty}((-\infty, 0])$ of (27) with $\lim _{\xi \rightarrow-\infty} U_{c}(\xi)=0$ and the following properties:

$$
\left\{\begin{array}{l}
\text { (i). } U_{c}^{\prime}(\xi)>0 \quad \text { for } \xi \in(-\infty, 0), \\
\text { (ii). } U_{c}^{\prime}(0)=0, \\
\text { (iii). } U_{c}^{\prime}(\xi) \leq \lambda_{c} U(\xi) \quad \text { for } \xi \in(-\infty, 0],
\end{array}\right.
$$


where $\lambda_{c}>0$ is a large constant depending on $c$ (and also on $p$ ). As a function of $c>0, U_{c}(0)$ is strictly decreasing, and given any $\delta>0$ and $A>0$, one can choose $c=c(\delta, A)>0$ so small that

$$
U_{c}(0)>\delta^{-1} \quad \text { and } \quad U_{c}^{\prime}(\xi)>A \quad \text { whenever } \delta \leq U_{c}(\xi) \leq \delta^{-1} .
$$

Proof. It will be convenient to look at $H=U^{p}>0$ instead of $U$ itself. We have $U=H^{1 / p}$, and (27) is equivalent to

$$
H H_{\xi \xi}=-c H_{\xi}+\frac{p-1}{p}\left(H_{\xi}\right)^{2}-p H^{2}+p H, \quad H(\xi)=U^{p}(\xi),
$$

which can be written as the first order system (let $\left.G=H_{\xi}\right)$

$$
\left\{\begin{array}{l}
H H_{\xi}=H G \\
H G_{\xi}=-p H^{2}+p H-c G+\frac{p-1}{p} G^{2} .
\end{array}\right.
$$

Thus up to a reparametrization (since there is a factor $H$ in front of $H_{\xi}$ and $G_{\xi}$ ), the positive solutions of (27) are in one-to-one correspondence with the orbits of the vector field

$$
X_{c}(H, G)=\left(H G,-p H^{2}+p H-c G+\frac{p-1}{p} G^{2}\right)
$$

lying on the region $R^{+}=\{(H, G): H>0\}$. We shall analyze the phase portrait of the vector field $X_{c}(H, G)$ in $R^{+}$. Note that $X_{c}$ has three zeros, $(0,0),(1,0)$, and $(0, p c /(p-1))$, in $R^{+}$. For a given small $c>0$, our aim is to look at a certain special solution $(H(\xi), G(\xi))$ of the system (33) with $H(\xi)>0$ everywhere.

If we compute the linearization of $X_{c}(H, G)$ at these equilibrium points, we obtain the three matrices

$$
M=\left(\begin{array}{cc}
G & H \\
p-2 p H & -c+\frac{2(p-1)}{p} G
\end{array}\right)=\left(\begin{array}{cc}
0 & 0 \\
p & -c
\end{array}\right), \quad\left(\begin{array}{cc}
1 \\
-p & -c
\end{array}\right), \quad\left(\begin{array}{cc}
\frac{p c}{p-1} & 0 \\
p & c
\end{array}\right)
$$

at $(0,0),(1,0)$ and $(0, p c /(p-1))$, respectively. The eigenvalues of them are given respectively by

$$
\lambda=0,-c, \quad \lambda=\frac{-c \pm \sqrt{c^{2}-4 p}}{2}, \quad \lambda=\frac{p c}{p-1}, c,
$$

where $c>0$ is a constant to be chosen later. Therefore, $(0,0)$ is a degenerate zero of $X_{c}$ (it is not a hyperbolic fixed point of $\left.X_{c}\right),(1,0)$ is a spiraling sink of $X_{c}$ if $c>0$ is such that $c^{2}-4 p<0$, and $(0, p c /(p-1))$ is a source of $X_{c}$.

By definition, the unstable set $W^{u}(O)$ of the origin $O=(0,0)$ consists of all orbits of $X_{c}$ which tend to $O$ as $\xi \rightarrow-\infty$. As the origin is degenerate, one needs to analyze further to know what $W^{u}(O)$ looks like. Note that if $U_{c}(\xi)$ is the solution satisfying Theorem 14, then

$$
\left(H_{c}(\xi), G_{c}(\xi)\right)=\left(U_{c}^{p}(\xi), p U_{c}^{p-1}(\xi) U_{c}^{\prime}(\xi)\right)
$$

parametrizes a trajectory of $X_{c}$ in the unstable set $W^{u}(O)$ of the origin. Thus one needs to look at $W^{u}(O)$.

Existence of a trajectory in $W^{u}(O)$. Given a constant $\lambda>0$, let $l_{\lambda}$ be the half line $G=\lambda H, H \geq 0$. The half line $l_{\lambda}$ has upward normal $(-\lambda, 1)$, and along it 
we have

$$
\left\langle X_{c},(-\lambda, 1)\right\rangle=\left\{p-c \lambda-\left(p+\frac{\lambda^{2}}{p}\right) H\right\} H, \quad \lambda>0, \quad H \geq 0 .
$$

Now choose two positive $\lambda_{1}, \lambda_{2}$ such that $\lambda_{1}<p / c$ and $\lambda_{2}=p / c$. Put $h^{*}=$ $\left(p-c \lambda_{1}\right) /\left(p+\lambda_{1}^{2} / p\right)>0$, and define the points

$$
A=\left(h^{*}, \lambda_{1} h^{*}\right), \quad B=\left(h^{*}, \lambda_{2} h^{*}\right), \quad O=(0,0) .
$$

Along the segment $O A$ with $0 \leq H \leq h^{*}$, we have $\left\langle X_{c},\left(-\lambda_{1}, 1\right)\right\rangle \geq 0$ (=0 only at $H=0$ or $H=h^{*}$ ), and so the vector field $X_{c}$, when restricted to $O A$, is pointing toward the upper half of segment $O A$. Similarly, along the segment $O B$ we have $\left\langle X_{c},\left(-\lambda_{2}, 1\right)\right\rangle \leq 0(=0$ only at $H=0)$. Hence the vector field $X_{c}$, when restricted to $O B$, is pointing toward the lower half of the segment.

Finally, along the segment $A B$, we have $\left\langle X_{c},(1,0)\right\rangle=h^{*} G>0$ for all $G$ with $\lambda_{1} h^{*}<G<\lambda_{2} h^{*}$. We conclude that the trajectories of $X_{c}$ enter the triangle $O A B$ through the sides $O A$ and $O B$, and they leave $O A B$ through the vertical side $A B$.

For any point $(H, G)$ inside the triangle $O A B$, it has the form $G=\lambda H$, for some $\lambda_{1} \leq \lambda \leq \lambda_{2}=p / c$, and hence

$$
\begin{aligned}
G_{\xi} & =\frac{1}{H}\left(-p H^{2}+p H-c \lambda H+\frac{p-1}{p} \lambda^{2} H^{2}\right) \\
& =(p-c \lambda)+\left(\frac{p-1}{p} \lambda^{2}-p\right) H \geq\left(\frac{p-1}{p} \lambda_{1}^{2}-p\right) H .
\end{aligned}
$$

We may choose $c$ and $\lambda_{1}$ such that $p / \sqrt{p-1}<\lambda_{1}<\lambda_{2}=p / c$ and conclude that

$$
G_{\xi} \geq\left(\frac{p-1}{p} \lambda_{1}^{2}-p\right) H>0
$$

for all $\lambda_{1} \leq \lambda \leq \lambda_{2}$ and $0<H \leq h^{*}=\left(p-c \lambda_{1}\right) /\left(p+\lambda_{1}^{2} / p\right)$. Therefore $G_{\xi}>0$ in the interior of the triangle $O A B$, and it follows from Wazewski's Principle that at least one of the trajectories through $A B$ tends to the origin as $\xi \rightarrow-\infty$.

In conclusion, we see that as long as $c>0$ is small enough (depending only on $p)$, there exists a trajectory in the unstable set $W^{u}(O)$.

Uniqueness of the trajectory in $W^{u}(O)$. Express the second equation of (33) as

$$
H G_{\xi}=-p\left(H-\frac{1}{2}\right)^{2}+\frac{p-1}{p}\left(G-\frac{p c}{2(p-1)}\right)^{2}+\frac{p}{4}\left(1-\frac{c^{2}}{p-1}\right) .
$$

We see that if $c<\sqrt{p-1}$, then the set $\left\{(H, G): H>0, G_{\xi}=0\right\}$ is the part of the hyperbola

$$
\Gamma: p\left(H-\frac{1}{2}\right)^{2}-\frac{p-1}{p}\left(G-\frac{p c}{2(p-1)}\right)^{2}=\frac{p}{4}\left(1-\frac{c^{2}}{p-1}\right)
$$

lying in $R^{+}=\{(H, G): H>0\}$. Here $\Gamma$ is centered at $(1 / 2, p c /(2(p-1)))$ and passes through the three equilibrium points $(0,0),(0, p c /(p-1))$ and $(1,0)$.

Consider the region $\Omega$ enclosed by the segment $\{(0, G): 0 \leq G \leq p c /(p-1)\}$ and the left branch of $\Gamma$. For each point $(H, G)$ inside $\Omega$, the vector field $X_{c}(H, G)=$ $\left(H H_{\xi}, H G_{\xi}\right)$ satisfies $H H_{\xi}=H G>0$ and $H G_{\xi}<0$. This implies that any trajectory $(H, G)$ in $W^{u}(O)$ will not pass through the region $\Omega$, and thus there exists a 
large constant $\lambda_{c}$ (say $\lambda_{c}>p / c$, where $p / c$ is the slope of $\Gamma$ at the origin) such that any trajectory $(H(\xi), G(\xi))$ in $W^{u}(O)$ satisfies $G \leq \lambda_{c} H$, as $\xi \rightarrow-\infty$.

Let $\left(H_{1}, G_{1}\right)$ and $\left(H_{2}, G_{2}\right)$ be two different orbits in $W^{u}(O)$. From the above observation, near the origin they can be represented as the graphs $G_{1}=g_{1}(H)$, $G_{2}=g_{2}(H)$, where the $g_{i}$ are solutions of the equation

$$
g^{\prime}(H)=\frac{H G_{\xi}}{H H_{\xi}}=\frac{-p H^{2}+p H-c G+\frac{p-1}{p} G^{2}}{H G}=\frac{(p-1) g(H)-p c}{p H}+\frac{p-p H}{g(H)} .
$$

Orbits cannot intersect, so we may assume that $g_{1}(H)<g_{2}(H)$. Their difference $w(H)=g_{2}(H)-g_{1}(H)>0$ satisfies the equation

$$
w^{\prime}(H)=\left(\frac{p-1}{p H}-\frac{p-p H}{g_{2}(H) g_{1}(H)}\right) w, \quad w>0 .
$$

Now for $H>0$ sufficiently small, we have $g_{1}(H) \leq \lambda H, g_{2}(H) \leq \lambda H$ for some $\lambda$, and so

$$
w^{\prime}(H) \leq\left(\frac{p-1}{p H}-\frac{p-p H}{g_{2}(H) g_{1}(H)}\right) w=\frac{w}{p \lambda^{2} H^{2}}\left[\lambda^{2}(p-1) H+p^{2} H-p^{2}\right]<0
$$

for all sufficiently small $H>0$, which means that $w(H)>0$ is decreasing on some interval $[0, \varepsilon), \varepsilon>0$. However, by $\lim _{H \downarrow 0} w(H)=0$ we get a contradiction and must have $w(H) \equiv 0$. Thus the two solutions are in fact equal.

Remark 15. Since the trajectory $(H(\xi), G(\xi))$ in $W^{u}(O)$ is unique, in the above existence proof we can choose $\lambda_{1}$ as close to $p / c$ as possible. In particular, $(H(\xi), G(\xi))$ in $W^{u}(O)$ must satisfy

$$
\frac{p}{c}=\lim _{\xi \rightarrow-\infty} \frac{G(\xi)}{H(\xi)}=\lim _{\xi \rightarrow-\infty} \frac{p U^{p-1}(\xi) U^{\prime}(\xi)}{U^{p}(\xi)}=\lim _{\xi \rightarrow-\infty} \frac{p U^{\prime}(\xi)}{U(\xi)},
$$

which implies the asymptotic behavior

$$
\lim _{\xi \rightarrow-\infty} \frac{U^{\prime}(\xi)}{U(\xi)}=\frac{1}{c}
$$

Let $\left(H_{c}(\xi), G_{c}(\xi)\right)$ denote the trajectory whose existence and uniqueness have been established and let $U_{c}(\xi)$ be the corresponding function of $\xi$. That is, $U_{c}(\xi)=$ $H_{c}^{1 / p}(\xi)$. Recall that along any positive solution $U(\xi)$ of (27), we have $d E / d \xi=$ $-2 c\left(U^{\prime}\right)^{2} / U^{p} \leq 0$, where $E(\xi)$ is given by (29). It follows that the quantity

$$
E(H, G)=\frac{G^{2}}{p^{2}} H^{2 / p-2}+H^{2 / p}-\frac{2}{2-p} H^{2 / p-1}, \quad H=U^{p}, \quad G=H_{\xi}
$$

is strictly decreasing on orbits of $X_{c}$, except $c=0$ (when $c=0$, all orbits are closed curves). Thus $H^{2 / p}-2(2-p)^{-1} H^{2 / p-1}$, and therefore $H$, are bounded from above on any orbit of $X_{c}$. Furthermore, it also implies that $|G|$ is bounded.

Using the fact that $(1,0)$ is an attracting spiral point, one can show that any orbit (here we only care about those orbits with positive $H$ everywhere) converges to $(1,0)$ and winds around this point infinitely many times. In particular, any orbit will intersect the $H$-axis (to see this, just look at the vector field (34)). For the function $U_{c}(\xi)$, this means that it will converge to 1 as $\xi \rightarrow \infty$ and that it will oscillate infinitely often around its limit value. Its derivative $U_{c}^{\prime}(\xi)$ must therefore vanish infinitely often; by replacing $U_{c}(\xi)$ by $U_{c}\left(\xi-\xi_{0}\right)$ for some $\xi_{0} \in \mathbb{R}$ 
if necessary, we may assume that the first zero of $U_{c}^{\prime}$ is $\xi=0$ and $U_{c}^{\prime}(\xi)>0$ for all $\xi \in(-\infty, 0)$.

So far we have constructed the solution $U_{c}(\xi)$ satisfying (i) and (ii) of (31). Since we also have (37), one can choose a large constant $\lambda_{c}>0$ so that (iii) of (31) is also satisfied for such $U_{c}(\xi)$.

This completes our construction of $U_{c}$. To finish the proof, we need to verify (32) for $U_{c}(\xi)$.

We observe that the segment of $W^{u}(O)$ which lies in the first quadrant is the graph of some function $G=g_{c}(H)$ for $0 \leq H \leq h_{c}$, where $\left(h_{c}, 0\right)$ is the first point of intersection of $W^{u}(O)$ with the $H$-axis. Since $U_{c}(0)=h_{c}^{1 / p}$, we have to show that $h_{c}$ is monotone decreasing in $c$.

Let $c^{\prime}<c$ be given, and suppose that $h_{c^{\prime}} \leq h_{c}$. We want to derive a contradiction. First assume that $h_{c^{\prime}}<h_{c}$. Compare the two vector fields $X_{c}$ and $X_{c^{\prime}}$ in the first quadrant. If the backward orbit of $X_{c}$ through $\left(h_{c}, 0\right)$ and the backward orbit of $X_{c^{\prime}}$ through $\left(h_{c^{\prime}}, 0\right)$ intersect at a first point $\left(H_{0}, G_{0}\right), G_{0}>0$, we have the following comparison of the two vector fields at $\left(H_{0}, G_{0}\right)$ :

$$
-p H_{0}^{2}+p H_{0}-c G_{0}+\frac{p-1}{p} G_{0}^{2}<-p H_{0}^{2}+p H_{0}-c^{\prime} G_{0}+\frac{p-1}{p} G_{0}^{2},
$$

which implies that the backward orbit of $X_{c^{\prime}}$ through $\left(h_{c^{\prime}}, 0\right)$ cannot pass through the graph of $g_{c}(H)$. As a consequence, the graph of $g_{c^{\prime}}(H)$ on the domain $0<$ $H<h_{c^{\prime}}$ must be below the graph of $g_{c}(H)$. On the other hand, by (37) we know

$$
g_{c}^{\prime}(0)=\lim _{\xi \rightarrow-\infty} \frac{g_{c}(H(\xi))}{H(\xi)}=\lim _{\xi \rightarrow-\infty} \frac{G(\xi)}{H(\xi)}=\frac{p}{c},
$$

and similarly $g_{c^{\prime}}^{\prime}(0)=p / c^{\prime}$. Hence $g_{c^{\prime}}^{\prime}(0)>g_{c}^{\prime}(0)$, and this gives a contradiction

$$
\lim _{H \rightarrow 0^{+}} g_{c^{\prime}}^{\prime}(H)=\frac{p}{c^{\prime}}>\lim _{H \rightarrow 0^{+}} g_{c}^{\prime}(H)=\frac{p}{c}>0 .
$$

Thus $h_{c^{\prime}}<h_{c}$ is impossible.

If $c^{\prime}<c$ but $h_{c^{\prime}}=h_{c}$, then by continuity we must have $0<g_{c^{\prime}}(H) \leq g_{c}(H)$ for all $0<H<h_{c^{\prime}}$. But now estimate (39) still holds and we obtain the same contradiction.

Remark 16. By (38) and (37), we have

$$
\begin{aligned}
\frac{p}{c} & =g_{c}^{\prime}(0)=\lim _{H \rightarrow 0^{+}} g_{c}^{\prime}(H)=\lim _{\xi \rightarrow-\infty} \frac{G_{\xi}}{H_{\xi}} \\
& =\lim _{\xi \rightarrow-\infty} \frac{p U^{p-1}(\xi) U^{\prime \prime}(\xi)+p(p-1) U^{p-2}(\xi)\left(U^{\prime}(\xi)\right)^{2}}{p U^{p-1}(\xi) U^{\prime}(\xi)}=\lim _{\xi \rightarrow-\infty} \frac{U^{\prime \prime}(\xi)}{U^{\prime}(\xi)}+\frac{p-1}{c}
\end{aligned}
$$

and derive the limit

$$
\lim _{\xi \rightarrow-\infty} \frac{U^{\prime \prime}(\xi)}{U^{\prime}(\xi)}=\frac{1}{c}
$$

From (37) and (40), it is not hard to see that asymptotically $U(\xi)$ is given by $a e^{(1 / c) \xi}$ as $\xi \rightarrow-\infty$ for some constant $a>0$.

A similar argument also shows that $g_{c}(H)$ is a strictly decreasing function of $c$ for fixed $H$, i.e., as $c \downarrow 0$, the unstable set $W_{c}^{u}(O)$ moves upwards.

We next claim that $U_{c}(0) \rightarrow \infty$ as $c \downarrow 0$. Assume that $U_{c}(0)$ were bounded, as $c \downarrow 0$. Then the $h_{c}$ 's would converge to some $h_{0}>1$. The vector field $X_{c}$ is 
well-defined and smooth for all $c \in \mathbb{R}$, so the unstable set $W_{c}^{u}(O)$, being the orbit of $X_{c}$ through $\left(h_{c}, 0\right)$, must converge to the orbit of $X_{0}$ through $\left(h_{0}, 0\right)$, where

$$
X_{0}(H, G)=\left(H G,-p H^{2}+p H+\frac{p-1}{p} G^{2}\right)
$$

and the quantity

$$
E(H, G)=\frac{G^{2}}{p^{2}} H^{2 / p-2}+H^{2 / p}-\frac{2}{2-p} H^{2 / p-1} \quad\left(H=U^{p}, G=p U^{p-1} U_{\xi}\right)
$$

is constant on the orbits of $X_{0}$. By (30) we know that all orbits of $X_{0}$ are periodic (due to the condition $p>2$ ). In particular, for $c=0$, the orbit of $X_{0}$ through $\left(h_{0}, 0\right)$ will satisfy the equation

$$
\frac{G^{2}}{p^{2}} H^{2 / p-2}+H^{2 / p}-\frac{2}{2-p} H^{2 / p-1}=h_{0}^{2 / p}-\frac{2}{2-p} h_{0}^{2 / p-1}>0, \quad h_{0}>1,
$$

and from this equation we see that the orbit of $X_{0}$ through $\left(h_{0}, 0\right)$ will intersect the $H$-axis at some point $\left(h_{*}, 0\right), 0<h_{*}<1$, when followed backwards in time, where $h_{*}$ satisfies

$$
h_{*}^{2 / p}-\frac{2}{2-p} h_{*}^{2 / p-1}=h_{0}^{2 / p}-\frac{2}{2-p} h_{0}^{2 / p-1} .
$$

By continuous dependence on parameters, the same will be true for some small $c>0$, a contradiction. Therefore we have $\lim _{c \downarrow 0} U_{c}(0)=\infty$.

Recall that for fixed $c>0$, the quantity

$$
E(H(\xi), G(\xi))=\frac{G^{2}(\xi)}{p^{2}} H^{2 / p-2}(\xi)+H^{2 / p}(\xi)-\frac{2}{2-p} H^{2 / p-1}(\xi)
$$

is strictly decreasing along the unstable orbit. We already know that as $c \downarrow 0$, $h_{c}(0) \uparrow \infty$. In particular, $E(H(\xi), G(\xi))$ is uniformly large on $(-\infty, 0]$ since

$$
E(H(0), G(0))=h_{c}^{2 / p}(0)-\frac{2}{2-p} h_{c}{ }^{2 / p-1}(0) \rightarrow \infty \quad \text { as } c \downarrow 0 .
$$

Now when we confine ourselves to the region $\delta \leq H=U^{p} \leq \delta^{-1}$, as $c \downarrow 0$, we must have $g_{c}(H)=G \uparrow \infty$ as $c \downarrow 0$, and uniformly so on the interval $\delta \leq H \leq 1 / \delta$.

Since $g_{c}(H)=G=p U_{c}^{p-1} U_{c}^{\prime}$ and $\delta \leq H \leq 1 / \delta$, we must have $U_{c}^{\prime}$ sufficiently large as $c \downarrow 0$. Therefore (32) also holds. The proof of Theorem 14 is done.

3.2. Proof of Theorem 10, Assume $v(x, t)$ is a type-one solution to equation (\$) with $p \geq 2$. Then the rescaled positive function $u(x, \tau)$ is bounded from above. Choose a large constant $A$ so that

$$
\left\{\begin{array}{l}
u(x, \tau) \leq A \text { for all }(x, \tau) \in S_{m}^{1} \times[0, \infty), \\
\left|u_{x}(x, 0)\right| \leq A \text { for all } x \in S_{m}^{1} \\
u(x, 0) \geq \frac{1}{A} \text { for all } x \in S_{m}^{1} .
\end{array}\right.
$$

Also choose $c>0$ so small that the solution $U_{c}(\xi)$ of the last section satisfies $U_{c}(0)>A$ and $U_{c}^{\prime}(\xi)>A$ whenever $A^{-1} \leq U_{c}(\xi) \leq A$. By Theorem 14 such a $c$ exists, together with the existence of a large constant $\lambda_{c}>0$ such that $0<U_{c}^{\prime}(\xi) \leq$ $\lambda_{c} U_{c}(\xi)$ for all $\xi \in(-\infty, 0]$. Note that here the number $c$ and $\lambda_{c}$ both depend on the initial data $u(x, 0)$. 
For any fixed $\left(x_{0}, \tau_{0}\right)$ we have $0<u\left(x_{0}, \tau_{0}\right)<U_{c}(0)$, and since $U_{c}(\xi)$ is strictly increasing on $(-\infty, 0]$ there exists a unique $x_{1} \in \mathbb{R}$ for which $U_{c}\left(x_{1}-c \tau_{0}\right)=$ $u\left(x_{0}, \tau_{0}\right)$. Consider the function

$$
u^{*}(x, \tau)=U_{c}\left(x-x_{0}+x_{1}-c \tau\right) .
$$

Then $u^{*}(x, \tau)$ is a solution of the equation $\partial u / \partial \tau=u^{p}\left(u_{x x}+u-u^{1-p}\right)$ on the region

$$
Q=\left\{(x, \tau): x<x_{0}-x_{1}+c \tau, \tau>0\right\}
$$

and the difference

$$
w(x, \tau)=u^{*}(x, \tau)-u(x, \tau)=U_{c}\left(x-x_{0}+x_{1}-c \tau\right)-u(x, \tau)
$$

satisfies a linear parabolic PDE of the form (see [ANG], p. 608)

$$
\frac{\partial w}{\partial \tau}=a(x, \tau) w_{x x}+b(x, \tau) w_{x}+c(x, \tau) w .
$$

We note that $w\left(x_{0}, \tau_{0}\right)=0$, and on the boundary $\partial Q \bigcap\{\tau>0\}$ (i.e., when $x=$ $\left.x_{0}-x_{1}+c \tau\right)$ we have

$$
w(x, \tau)=U_{c}(0)-u(x, \tau) \geq U_{c}(0)-A>0 .
$$

On the other part of $\partial Q$, i.e., when $x<x_{0}-x_{1}$ and $\tau=0$ we have

$$
w\left(x_{0}-x_{1}, 0\right)=U_{c}(0)-u\left(x_{0}-x_{1}, 0\right)>0,
$$

and $w(x, 0)=U_{c}\left(x-x_{0}+x_{1}\right)-u(x, 0)$ becomes negative as $x \rightarrow-\infty$, due to (41). Hence $w(x, 0)$ must have at least one zero $y_{0}$ on $\left(-\infty, x_{0}-x_{1}\right)$. At any zero $y_{0}$ we have

$$
\frac{1}{A} \leq u\left(y_{0}, 0\right)=U_{c}\left(y_{0}-x_{0}+x_{1}\right) \leq A
$$

and so

$$
w_{x}\left(y_{0}, 0\right)=U_{c}^{\prime}\left(y_{0}-x_{0}+x_{1}\right)-u_{x}\left(y_{0}, 0\right)>A-u_{x}\left(y_{0}, 0\right) \geq 0 .
$$

Hence $w(x, 0)$ cannot have more than one zero on the interval $\left(-\infty, x_{0}-x_{1}\right)$.

By the Sturmian theorem, the number of zeros of $x \rightarrow w(x, \tau)$, counted with multiplicity, cannot increase with time. Now by our construction we have $w\left(x_{0}, \tau_{0}\right)=0$, and since this is the only zero of $w\left(\cdot, \tau_{0}\right)$, we must have $w_{x}\left(x_{0}, \tau_{0}\right)>0$ (since $w\left(x_{0}-x_{1}+c \tau_{0}, \tau_{0}\right)>0$ and $\left.w\left(-\infty, \tau_{0}\right)<0\right)$. Thus

$$
u_{x}\left(x_{0}, \tau_{0}\right)<U_{c}^{\prime}\left(x_{1}-c \tau_{0}\right) \leq \lambda_{c} U_{c}\left(x_{1}-c \tau_{0}\right)=\lambda_{c} u\left(x_{0}, \tau_{0}\right) .
$$

By applying the same argument to $u(-x, \tau)$ one can also obtain $-u_{x} \leq \lambda_{c} u$ so that $\left|u_{x}(x, \tau)\right| \leq \lambda_{c} u(x, \tau)$ for all $(x, \tau) \in \in S_{m}^{1} \times[0, \infty)$. The proof of Theorem 10 is done.

3.3. Proof of type-one convergence. To go further we need to look more closely at the following ODE:

$$
w^{\prime \prime}(x)+w(x)-w^{1-p}(x)=0, \quad x \in(-\infty, \infty), \quad p>2 .
$$

It is easy to see that any solution $w(x)$ to it is positive everywhere and periodic over $x \in(-\infty, \infty)$ (this property is valid for $p \geq 2$; when $p \in(0,2), w(x)$ may have different behavior; see [LPT] and $[\mathrm{PT}])$. Let $a \leq 1$ be the minimal value of 
$w(x)$ on $(-\infty, \infty)$. Without loss of generality, we may assume that $a=w(0)$ (and so $w^{\prime}(0)=0$ ), and by reflection (if $w(x)$ is a solution, so is $w(-x)$ ) $w(x)$ must be symmetric with respect to any local maximum point or minimum point. It also satisfies the energy identity

$$
\left(w^{\prime}(x)\right)^{2}+w^{2}(x)-\frac{2}{2-p} w^{2-p}(x)=F(a) \quad \text { for all } \quad x \in(-\infty, \infty),
$$

where $F$ is the positive function $F(s)=s^{2}-2(2-p)^{-1} s^{2-p}$ defined on $s \in$ $(0, \infty)$. For $p>2$, the convex positive function $F(s)$ decreases on $s \in(0,1)$ with $\lim _{s \rightarrow 0^{+}} F(s)=+\infty$ and increases to $+\infty$ on $(1, \infty)$. Given $a \in(0,1]$, there is a unique $b \geq 1$ so that $F(a)=F(b)$, where $b=\max _{x \in \mathbb{R}} w(x)$, and the minimal period $T=2 R(a)$ of $w(x)$ is given by

$$
\begin{aligned}
T=2 R(a) & =2 \int_{a}^{b} \frac{d s}{\sqrt{F(a)-F(s)}} \\
& =2 \int_{a}^{b} \frac{d s}{\sqrt{\left(a^{2}-\frac{2}{2-p} a^{2-p}\right)-\left(s^{2}-\frac{2}{2-p} s^{2-p}\right)}}, \\
F(b) & =F(a) .
\end{aligned}
$$

The above integral is improper near both $a$ and $b$.

It has been shown in Urbas U2 that

$$
\lim _{a \rightarrow 0^{+}} R(a)=\frac{\pi}{2}, \quad \lim _{a \rightarrow 1^{-}} R(a)=\frac{\pi}{\sqrt{p}}, \quad p \in(2, \infty) .
$$

Moreover, by Corollary 5.6 of Andrews [AN3, we know that $R(a)$ is strictly decreasing in $a \in(0,1)$ when $p \in(4, \infty)$ and strictly increasing in $a \in(0,1)$ when $p \in(2,4)$. When $p=4$, all solutions of equation (45) are $\pi$-periodic (see (2) also).

Remark 17. As a comparison, when $p \in(0,2)$ we have (see [U2] and [AN3] again)

$$
\lim _{a \rightarrow 0^{+}} R(a)=\frac{\pi}{p}, \quad \lim _{a \rightarrow 1^{-}} R(a)=\frac{\pi}{\sqrt{p}}, \quad p \in(0,2),
$$

and $R(a)$ is strictly decreasing in $a \in(0,1)$ when $p \in(0,1)$ and strictly increasing in $a \in(0,1)$ when $p \in(1,2)$. When $p=1$, all solutions to the ODE (45) have period $2 \pi$.

One can also write the ODE (45) as a system

$$
\frac{d w}{d x}=h, \quad \frac{d h}{d x}=-w+w^{1-p}, \quad p>2 .
$$

Then the vector field $V(w, h)=\left(h,-w+w^{1-p}\right)$ has only one equilibrium point $(1,0)$ on the half-plane $\{w>0\}$, and the eigenvalues of the linearization at it are $\lambda= \pm \sqrt{p} i$ (this matches with the second limit of (48)). The phase portrait of $V$ on $\{w>0\}$ is a family of closed orbits $C(a)$ centered at $(1,0)$ with period $2 R(a)$, where $a=\min _{x \in \mathbb{R}} w(x)$. Thus the intersections of $C(a)$ and the $w$-axis are $(a, 0)$ and $(b, 0)$ with $F(a)=F(b), w(0)=a \leq 1, w(R(a))=b \geq 1, w^{\prime}(0)=w^{\prime}(R(a))=0$. 
We can now state the following convergence theorem, which is a generalization of Theorem A of [ANG]:

Theorem 18 (Convergence of type-one blow-up for $p>2$ ). Let $p>2$ and let $v(x, t)>0$ be a type-one solution of (\$) defined on some maximal time interval $\left[0, T_{\max }\right)$. Then as $\tau \rightarrow \infty$ the rescaled solution $u(x, \tau)$, given by (20), converges in $C^{\infty}\left(S_{m}^{1}\right)$ to a smooth positive $2 m \pi$-periodic function $w(x)$, which is an entire solution of the $O D E$

$$
w^{\prime \prime}(x)+w(x)-w^{1-p}(x)=0 \quad \text { for all } x \in \mathbb{R} .
$$

Remark 19. See Andrews [AN3 for a complete classification of solutions to ODE (50).

Proof. Assume a type-one blow-up of $v(x, t)$. For any sequence $\tau_{n} \rightarrow \infty$, by the Arzela-Ascoli theorem there is a subsequence, which we also call $\tau_{n}$, so that $u\left(x, \tau_{n}\right)$ converges uniformly on $S_{m}^{1}$ to a Lipschitz function $w(x) \geq 0$, which is $2 m \pi$-periodic. By Theorem 10, $u(x, \tau)$ has positive lower bound $e^{-2 \lambda m \pi}$ for all $\tau$ (see (25D), hence $w(x)$ is strictly positive everywhere. Now we can apply a similar argument as in Propositions 12 and 14 of [LPT] (since $p>2, u(x, \tau)$ has a positive lower bound is essential in (32), p. 160 of [LPT] $)$ to obtain the conclusion that $w(x)$ satisfies the ODE (50) everywhere. By regularity theory for uniform parabolic equations, $w(x)$ is smooth and we have $C^{\infty}$ convergence of $u\left(x, \tau_{n}\right)$ to $w(x)$ as $\tau_{n} \rightarrow \infty$.

If we do not have full time convergence of $u(x, \tau)$ as $\tau \rightarrow \infty$, then there will exist two sequences of time $\tau_{n} \rightarrow \infty$ and $\tilde{\tau}_{n} \rightarrow \infty$ such that $u\left(x, \tau_{n}\right) \rightarrow w(x)$ and $u\left(x, \tilde{\tau}_{n}\right) \rightarrow \tilde{w}(x)$, where $w, \tilde{w}$ are different positive $2 m \pi$-periodic solutions of the ODE (50). Let $a, \tilde{a} \in(0,1]$ be the minimum values of $w, \tilde{w}$. We may assume $a \leq \tilde{a}$. Note that although $w(x)$ is different from $\tilde{w}(x)$, it may be possible to have $a=\tilde{a}$. By the above discussion, we have $w(R(a))=b \geq \tilde{w}(R(\tilde{a}))=\tilde{b} \in[1, \infty)$.

If we have $a=\tilde{a}$, then $w(x)$ must be a translation of $\tilde{w}(x)$ and we can find some $x_{0} \in \mathbb{R}$ with $w^{\prime}\left(x_{0}\right) \tilde{w}^{\prime}\left(x_{0}\right)<0$ (i.e., they have different signs). This would contradict Proposition 23 of [LPT]. Therefore we only have to consider the case $a<\tilde{a}$.

For $a<\tilde{a}$ there are two cases to discuss.

Case 1. $p \in(2, \infty), p \neq 4$.

By the discussion before Remark 17, in such a case we must have $R(a) \neq R(\tilde{a})$ since $R(a)$ is a monotone function in $a \in(0,1)$. Now $w(x)$ and $\tilde{w}(x)$ have different periods, and we can find some $x_{0} \in \mathbb{R}$ such that $w^{\prime}\left(x_{0}\right) \tilde{w}^{\prime}\left(x_{0}\right)<0$. We obtain the same contradiction due to Proposition 23 of [LPT].

Case 2. $p=4$.

In this case by (2), up to a translation, all solutions to the ODE $w^{\prime \prime}+w-w^{-3}=0$ are $\pi$-periodic (see [AN3, [U2]) and are given by (if $w(0) \geq 1$ is the maximum) the 1-parameter family of functions in (2). Unfortunately now for any $x_{0} \in \mathbb{R}$ we have $w^{\prime}\left(x_{0}\right) \tilde{w}^{\prime}\left(x_{0}\right) \geq 0$, and thus Proposition 23 of [LPT is not applicable here. A different method has to be used here 2

Let $\mathcal{Z}[w-\tilde{w}]$ denote the number of zero points $\xi \in S_{m}^{1}$ (or $\xi \in[-m \pi, m \pi)$ ) with $w(\xi)-\tilde{w}(\xi)=0$. Also let $Y$ denote the function space of all solutions of

\footnotetext{
${ }^{2}$ D.-H. Tsai would like to thank Professor Matano for teaching him the zero-number argument several years ago. It is now used in the proof of Theorem 18
} 
the ODE (50) on $\mathbb{R}$ (since now $p=4$, all solutions to the ODE (50) have minimal period $\pi)$. For any $\xi \in S_{m}^{1}$ with $w(\xi)-\tilde{w}(\xi)=0$, by uniqueness we must have $w^{\prime}(\xi) \neq \tilde{w}^{\prime}(\xi)$. Hence at each intersection point the graphs of the two functions $w, \tilde{w}$ are transversal.

For any $z(x) \in Y$, the difference $u(x, \tau)-z(x)$ satisfies a linear parabolic equation of the form (43) (since $z(x)$ is also a solution to the PDE (21)). By Angenent's result on p. 607 of [ANG] (Lemma 2.4 on p. 165 of Chen-Matano [CM] is more applicable here), the number $\mathcal{Z}[u(\cdot, \tau)-z(\cdot)]$ is nonincreasing in time $\tau \in(0, \infty)$. Also note that we have the convergence of $u\left(x, \tau_{n}\right)$ to $w(x)$ in $C^{1}$, which implies

$$
\mathcal{Z}\left[u\left(\cdot, \tau_{n}\right)-z(\cdot)\right]=\mathcal{Z}[w-\tilde{w}]
$$

for all large $n$ and all $z \in Y$ that are sufficiently close to $\tilde{w}$ in $C^{1}$ norm on $S_{m}^{1}$. In particular, we can conclude the following: there exists a time $T>0$ and a number $\delta>0$ such that

$$
\mathcal{Z}[u(\cdot, \tau)-z(\cdot)]=\mathcal{Z}[w-\tilde{w}]
$$

for all $\tau>T$ and all $z \in Y$ satisfying $\|z-\tilde{w}\|_{C^{1}\left(S_{m}^{1}\right)}<\delta$.

The fact that the number $\mathcal{Z}[u(\cdot, \tau)-z(\cdot)]$ remains a constant for large time implies that the function $x \rightarrow u(x, \tau)-z(x)$ does not have a degenerate zero (i.e., multiple zero) in $S_{m}^{1}$ for any fixed $\tau>T$ (see [CM]). But since $u\left(x, \tilde{\tau}_{n}\right)$ converges to $\tilde{w}(x)$ in $C^{1}\left(S_{m}^{1}\right)$ norm as $n \rightarrow \infty$, the graph of the function $x \rightarrow u\left(x, \tilde{\tau}_{n}\right)$ must be tangential to the graph of some $z \in Y$ satisfying $\|z-\tilde{w}\|_{C^{1}\left(S_{m}^{1}\right)}<\delta$. For example, for fixed $x_{0}$ one can choose $z(x)$ to be the solution of

$$
\left\{\begin{array}{l}
z^{\prime \prime}(x)+z(x)-z^{-3}(x)=0 \\
z\left(x_{0}\right)=u\left(x_{0}, \tilde{\tau}_{n}\right), \quad z^{\prime}\left(x_{0}\right)=u_{x}\left(x_{0}, \tilde{\tau}_{n}\right)
\end{array}\right.
$$

Then as $n$ large enough, $z(x)$ will be close to $\tilde{w}(x)$ in $C^{1}\left(S_{m}^{1}\right)$ since $u\left(x_{0}, \tilde{\tau}_{n}\right)$ is close to $\tilde{w}\left(x_{0}\right)$ and $u_{x}\left(x_{0}, \tilde{\tau}_{n}\right)$ is close to $\tilde{w}^{\prime}\left(x_{0}\right)$. Now $u\left(x, \tilde{\tau}_{n}\right)-z(x)$ has a degenerate zero at $x_{0}$, which is a contradiction.

Remark 20. Since $p=4$, any solution $z(x)$ to the ODE (52) has minimal period $\pi$. In particular, this implies that $z(x) \in C^{1}\left(S_{m}^{1}\right)$.

The above contradiction for either Case 1 or Case 2 implies that $w(x) \equiv \tilde{w}(x)$. The proof is done.

Theorem 18 implies that for the contracting flow $(\star)$, if $k_{\max }(t)\left(T_{\max }-t\right)^{1 /(\alpha+1)}$ remains bounded as $t \rightarrow T_{\max }$, then the rescaled curvature

$$
K(x, \tau)=\left(p^{1 / p} T_{\max }^{1 / p} e^{-\tau}\right)^{1 / \alpha} k\left(x, T_{\max }\left(1-e^{-p \tau}\right)\right), \quad \alpha \in(0,1], \quad \tau \in[0, \infty)
$$

converges in $C^{\infty}$ to a positive $K(x) \in C^{\infty}\left(S_{m}^{1}\right)$, which satisfies the ODE

$$
\left(K^{\alpha}\right)^{\prime \prime}(x)+K^{\alpha}(x)-\frac{1}{K(x)}=0 \quad \text { for all } \quad x \in \mathbb{R} .
$$

Geometrically this says that the evolving convex immersed closed curve $\gamma_{t}$ shrinks to a point in an asymptotically self-similar way. 


\section{TYPE-TWO BLOW-UP}

We now turn to the much more difficult type-one blow-up. We point out that in the proof of Theorem 10, the integral condition (3) does not come into play at all. Hence even if it is not satisfied, Theorem 10 still holds. In view of this, we have the following interesting observation:

Lemma 21 (Existence of type-two blow-up for $p \geq 2$ ). Assume $v_{0}(x)>0 \in$ $C^{\infty}\left(S_{m}^{1}\right)$ in (\$) does not satisfy (3) , i.e.,

$$
\int_{S_{m}^{1}} v_{0}^{1-p}(x) e^{i x} d x \neq 0 .
$$

Then we have type-two blow-up for the solution $v(x, t)$ to $(\mathbf{\beta})$, which means

$$
\limsup _{t \rightarrow T_{\max }}\left(v_{\max }(t)\left(T_{\max }-t\right)^{1 / p}\right)=\infty \text {. }
$$

Proof. Without loss of generality we may assume

$$
\int_{S_{m}^{1}} v_{0}^{1-p}(x) \cos x d x>0
$$

Since we have

$$
\int_{S_{m}^{1}} v^{1-p}(x, t) e^{i x} d x=\int_{S_{m}^{1}} v_{0}^{1-p}(x) e^{i x} d x
$$

for all $t \in\left[0, T_{\max }\right), u(x, \tau)$ satisfies

$$
\lim _{\tau \rightarrow \infty} \int_{S_{m}^{1}} u^{1-p}(x, \tau) \cos x d x=\lim _{\tau \rightarrow \infty}\left(p^{1 / p} T_{\max }^{1 / p} e^{-\tau}\right)^{1-p} \int_{S_{m}^{1}} v_{0}^{1-p}(x) \cos x d x=\infty
$$

which means that $\liminf _{\tau \rightarrow \infty} u_{\min }(\tau)=0$. If we have type-one blow-up, then Theorem 10 would imply a positive lower bound of $u_{\min }(\tau)$, a contradiction.

Remark 22. Thus for $p \geq 2$, type-two blow-up in equation (\$) is generic. Moreover, type-one blow-up occurs only when the initial data satisfies the integral condition (3).

Remark 23. When (3) is satisfied, then either type-one or type-two blow-up can happen. For type-one, just take a separable solution of (⿻) of the form $v(x, t)=$ $h(t) g(x)$, where $g(x)>0$ on $S_{m}^{1}$ satisfies the ODE (50) and $h(t)$ satisfies $d h / d t=$ $h^{1+p}, h(0)>0$. For type-two, choose a convex immersed plane curve with one big loop and one tiny loop. Then the corresponding evolution will become singular without shrinking to a point in an asymptotically self-similar way. Hence we obtain a type-two blow-up. The difficulty lies in the estimate of the blow-up rate.

4.1. A special symmetric case for type-two blow-up and convergence. In this section we assume the initial data $v_{0}(x)>0$ to equation (\$) satisfies (3) and the following symmetric condition:

$$
v_{0}(x)=v_{0}(-x) \quad \text { and } \quad v_{0}^{\prime}(x)<0, \text { for all } \quad x \in(0, m \pi) .
$$

If $v(x, t)$ is a solution to (o) with the above initial data $v_{0}(x)$, then $\tilde{v}(x, t):=$ $v(-x, t)$ is also a solution to $(\mathbf{\alpha})$ with $\tilde{v}(x, 0)=v_{0}(-x)=v_{0}(x)$ for all $x \in S_{m}^{1}$. By uniqueness we must have

$$
v(x, t)=v(-x, t) \quad \text { for all } \quad(x, t) \in(0, m \pi) \times\left[0, T_{\max }\right),
$$


which also implies

$$
v_{x}(0, t)=v_{x}(m \pi, t)=0 \quad \text { for all } t \in\left[0, T_{\max }\right) .
$$

Also the second condition of (57) implies that $v_{0}^{\prime}(x)$ has exactly two zeros on $S_{m}^{1}$, and since the number of zeros for $v_{x}(x, t)$ is nonincreasing in time, we must have

$$
v_{x}(x, t)<0 \text { for all }(x, t) \in(0, m \pi) \times\left[0, T_{\max }\right) .
$$

Hence the two conditions of (57) are preserved for all time. In particular, we have $v_{\max }(t)=v(0, t)$ for $t \in\left[0, T_{\max }\right)$.

The main result in this section is the following convergence behavior for type-two blow-up. One can view it as a partial generalization of Theorem $\mathrm{C}$ of ANG to the case $p \geq 2$ since here we assume $v_{0}(x)$ is symmetric and our convergence is only uniform, weaker than Angenent's $C^{\infty}$ convergence. However, the advantage of focusing on the symmetric case (57) is that we always have type-two blow-up and the proof of convergence in Theorem 25 below is very simple and straightforward.

Lemma 24. Assume $v(x, t)$ is a positive solution of $(\boldsymbol{\beta})$ in $S_{m}^{1}$ (with $m \geq 2$ ) where $v_{0}(x)$ satisfies (3) and (57). Then $v(x, t)$ has type-two blow-up.

Proof. Basically, we follow the arguments on p. 630 of ANG. If $v(x, t)$ has typeone blow-up, then by (25) we have

$$
v(x, t) \rightarrow \infty \text { as } t \rightarrow T_{\max } \text { for all } x \in[-m \pi, m \pi] .
$$

That is, the blow-up set of $v(x, t)$ is the whole domain.

When $m$ is even, $m=2 k, k \geq 1$, consider the function

$$
D(t)=\int_{0}^{(2 k-1) \pi} v^{1-p}(x, t) \cos x d x
$$

By (60), we have

$$
D(t) \rightarrow 0 \quad \text { as } \quad t \rightarrow T_{\max }
$$

Now by (\$) we compute

$$
\begin{aligned}
D^{\prime}(t) & =(1-p) \int_{0}^{(2 k-1) \pi}\left(v_{x x}(x, t)+v(x, t)\right) \cos x d x \\
& =\left.(1-p)\left(v(x, t) \sin x+v_{x}(x, t) \cos x\right)\right|_{0} ^{(2 k-1) \pi}=(p-1) v_{x}((2 k-1) \pi, t)<0
\end{aligned}
$$

due to (58) and (59). Hence $D(t)$ is decreasing, and by (61) it is positive for all $t \in\left[0, T_{\max }\right)$. Also the symmetry of $v(x, t)$ implies that

$$
\int_{0}^{2 k \pi} v^{1-p}(x, t) \cos x d x=\frac{1}{2} \int_{-2 k \pi}^{2 k \pi} v^{1-p}(x, t) \cos x d x=0 .
$$

Thus we have

$$
0=\int_{0}^{(2 k-1) \pi} v^{1-p}(x, t) \cos x d x+\int_{(2 k-1) \pi}^{2 k \pi} v^{1-p}(x, t) \cos x d x
$$

and then

$$
\int_{(2 k-1) \pi}^{2 k \pi} v^{1-p}(x, t) \cos x d x<0 \quad \text { for all } \quad t \in\left[0, T_{\max }\right) .
$$


However, by (59) we have

$$
\int_{(2 k-1) \pi}^{2 k \pi} v^{1-p}(x, t) \cos x d x>0 \text { for all } t \in\left[0, T_{\max }\right),
$$

which gives a contradiction.

When $m$ is odd, $m=2 k+1, k \geq 1$, we consider the function

$$
D(t)=\int_{0}^{2 k \pi} v^{1-p}(x, t) \cos x d x
$$

and again by (60), we have (61). Now

$$
\begin{aligned}
D^{\prime}(t) & =(1-p) \int_{0}^{2 k \pi}\left(v_{x x}(x, t)+v(x, t)\right) \cos x d x \\
& =\left.(1-p)\left(v(x, t) \sin x+v_{x}(x, t) \cos x\right)\right|_{0} ^{2 k \pi}=(1-p) v_{x}(2 k \pi, t)>0,
\end{aligned}
$$

and so $D(t)$ is increasing and therefore negative for all time. By symmetry again we obtain

$$
\int_{0}^{(2 k+1) \pi} v^{1-p}(x, t) \cos x d x=\frac{1}{2} \int_{-(2 k+1) \pi}^{(2 k+1) \pi} v^{1-p}(x, t) \cos x d x=0,
$$

and thus

$$
\int_{2 k \pi}^{(2 k+1) \pi} v^{1-p}(x, t) \cos x d x>0 \text { for all } t \in\left[0, T_{\max }\right) .
$$

However, by (59) we have

$$
\int_{2 k \pi}^{(2 k+1) \pi} v^{1-p}(x, t) \cos x d x<0 \text { for all } t \in\left[0, T_{\max }\right),
$$

which gives a contradiction.

Theorem 25 (Convergence of type-two blow-up for $p \geq 2$ with symmetric $v_{0}(x)$ ). Let $\Phi(x)=\cos x$ on $[-\pi / 2, \pi / 2]$ and $\Phi(x)=0$ otherwise. Assume $v_{0}(x)>0 \in$ $C^{\infty}\left(S_{m}^{1}\right)$ satisfies conditions (3) and (57). Then there exists a sequence of times $t_{n} \nearrow T_{\max }$ such that

$$
\lim _{n \rightarrow \infty} \frac{v\left(x, t_{n}\right)}{v\left(0, t_{n}\right)}=\Phi(x) \quad \text { uniformly on } \quad x \in[-m \pi, m \pi] .
$$

Remark 26. Note that if we have type-one blow-up, then we consider the rescaling $v(x, t) / R(t)$, where $R(t)$ is comparable to $v_{\max }(t)$. Hence here for type-two blowup, by analogy, it is reasonable to look at the rescaling $v(x, t) / v_{\max }(t)$, which is (62).

Proof. By Lemma 24, $v(x, t)$ has type-two blow-up, and so $\left(T_{\max }-t\right)^{1 / p} v_{\max }(t)$ is not bounded on $t \in\left[0, T_{\max }\right)$. Hence there exists a sequence $s_{n} \nearrow T_{\max }$ such that

$$
\lim _{n \rightarrow \infty}\left(T_{\max }-s_{n}\right)^{1 / p} v_{\max }\left(s_{n}\right)=\infty .
$$

Let

$$
\psi_{n}(x)=\frac{1}{T_{\max }-s_{n}} \int_{s_{n}}^{T_{\max }} \frac{v(x, s)}{v(0, s)} d s, \quad x \in[-m \pi, m \pi] .
$$


As we shall be interested in the behavior of $\psi_{n}(x)$ for $n$ large, without loss of generality, we may assume that $v_{\max }(t)=v(0, t)$ is increasing in time for all $t \in$ $\left[0, T_{\max }\right)$ (see Lemma 6) and by (16) we have

$$
0<\psi_{n}(x) \leq 1 \text { and }\left|\psi_{n}^{\prime}(x)\right| \leq 1 \quad \text { for all } \quad x \in[-m \pi, m \pi]
$$

for all $n$. We also have $\psi_{n}(x)=\psi_{n}(-x)$ for all $x \in[0, m \pi]$ and $n$. Moreover we have for all $n$ that

$$
\psi_{n}^{\prime}(x)<0 \text { for all } x \in(0, m \pi) .
$$

Let $0<K<\pi / 2$ be a fixed number but arbitrary. By (17) we know that when $t$ is close to $T_{\max }$, there holds

$$
v(x, t) \geq v_{\max }(t) \cos x=v(0, t) \cos x \quad \text { for all } \quad x \in[-K, K],
$$

and so

$$
\lim _{t \rightarrow T_{\max }} v(x, t)=\infty \text { for all } x \in[-K, K] .
$$

Moreover, by Lemma 6, we also have

$$
\left(v_{x x}+v\right)(x, t)>0, \quad x \in[-K, K]
$$

when $t$ is close to $T_{\max }$. As a consequence, when $n$ is large, we have

$$
\begin{aligned}
0 & <\frac{1}{T_{\max }-s_{n}} \int_{s_{n}}^{T_{\max }} \frac{v_{x x}(x, s)+v(x, s)}{v(0, s)} d s=\frac{1}{T_{\max }-s_{n}} \int_{s_{n}}^{T_{\max }} \frac{v_{s}(x, s)}{v^{p}(x, s) v(0, s)} d s \\
& \leq \frac{1}{T_{\max }-s_{n}} \int_{s_{n}}^{T_{\max }} \frac{v_{s}(x, s)}{v^{p+1}(x, s)} d s=\frac{1}{p\left(T_{\max }-s_{n}\right) v^{p}\left(x, s_{n}\right)} \text { for all } x \in[-K, K] .
\end{aligned}
$$

By (65), we may assume that $\psi_{n}(x)$ converges uniformly on $S_{m}^{1}$ to some $w(x) \in$ $C^{0}\left(S_{m}^{1}\right)$ and $w(x) \geq 0$ in $S_{m}^{1}$. For any test function $\varphi \in C_{0}^{\infty}(-\pi / 2, \pi / 2)$, choose $0<K<\pi / 2$ so that $(-K, K)$ contains the support of $\varphi$. By the Fubini theorem and integration by parts we have

$$
\begin{aligned}
& \int_{-\frac{\pi}{2}}^{\frac{\pi}{2}} w(x)\left[\varphi_{x x}(x)+\varphi(x)\right] d x=\lim _{n \rightarrow \infty} \int_{-K}^{K} \psi_{n}(x)\left[\varphi_{x x}(x)+\varphi(x)\right] d x \\
& =\lim _{n \rightarrow \infty} \int_{-K}^{K}\left[\left(\frac{1}{T_{\max }-s_{n}} \int_{s_{n}}^{T_{\max }} \frac{v(x, s)}{v(0, s)} d s\right)\left[\varphi_{x x}(x)+\varphi(x)\right]\right] d x \\
& =\lim _{n \rightarrow \infty} \frac{1}{T_{\max }-s_{n}} \int_{s_{n}}^{T_{\max }}\left[\int_{-K}^{K} \frac{v(x, s)}{v(0, s)}\left[\varphi_{x x}(x)+\varphi(x)\right] d x\right] d s \\
& =\lim _{n \rightarrow \infty} \frac{1}{T_{\max }-s_{n}} \int_{s_{n}}^{T_{\max }}\left[\int_{-K}^{K} \frac{v_{x x}(x, s)+v(x, s)}{v(0, s)} \varphi(x) d x\right] d s \\
& =\lim _{n \rightarrow \infty} \int_{-K}^{K}\left[\frac{1}{T_{\max }-s_{n}} \int_{s_{n}}^{T_{\max }} \frac{v_{x x}(x, s)+v(x, s)}{v(0, s)} d s\right] \varphi(x) d x=0
\end{aligned}
$$

due to (63), (67) and (69). This implies that $w(x)$ is a weak solution of the ODE $w_{x x}+w=0$ in $(-\pi / 2, \pi / 2)$ (note that since $\left|\psi_{n}^{\prime}(x)\right|$ is uniformly bounded, the function $w$ is Lipschitz continuous with $\left.w \in W^{1,2}\left(S_{m}^{1}\right)\right)$. Regularity theory implies 
that $w(x)$ is smooth in $x \in(-\pi / 2, \pi / 2)$ with $w_{x x}+w=0$. By our definition, $\psi_{n}(x)$ is decreasing in $x$ for $x \in(0, m \pi)$ and has a maximum at $x=0$ with $\psi_{n}(0)=1$. This implies that $w(x)$ is decreasing for $x \in(0, m \pi)$ and has a maximum at $x=0$. Hence $w(0)=1, w^{\prime}(0)=0$, and therefore $w(x)=\cos x$ for $x \in(-\pi / 2, \pi / 2)$.

By Lemma 7 we have for large $n$

$$
\begin{aligned}
0 & \leq \frac{1}{T_{\max }-s_{n}} \int_{s_{n}}^{T_{\max }} \int_{-K}^{K}\left(\frac{v(x, s)}{v(0, s)}-\cos x\right) d x d s \\
& =\int_{-K}^{K} \frac{1}{T_{\max }-s_{n}} \int_{s_{n}}^{T_{\max }}\left(\frac{v(x, s)}{v(0, s)}-\cos x\right) d s d x \\
& =\int_{-K}^{K}\left(\psi_{n}(x)-\cos x\right) d x \rightarrow 0 \text { as } n \rightarrow \infty .
\end{aligned}
$$

Hence if we let

$$
f(s)=\int_{-K}^{K}\left(\frac{v(x, s)}{v(0, s)}-\cos x\right) d x, \quad s \in\left[0, T_{\max }\right)
$$

we would have

$$
0 \leq \frac{1}{T_{\max }-s_{n}} \int_{s_{n}}^{T_{\max }} f(s) d s \rightarrow 0 \quad \text { as } \quad n \rightarrow \infty .
$$

Therefore by the mean value theorem we can find a sequence $s_{n}^{\prime}, s_{n}<s_{n}^{\prime}<T_{\max }$ so that

$$
\int_{-K}^{K}\left(\frac{v\left(x, s_{n}^{\prime}\right)}{v\left(0, s_{n}^{\prime}\right)}-\cos x\right) d x \rightarrow 0 \quad \text { as } \quad n \rightarrow \infty .
$$

Note that both $\cos x$ and $v\left(x, s_{n}^{\prime}\right) / v\left(0, s_{n}^{\prime}\right)$ are bounded functions with bounded derivatives (and their bounds are independent of $n$ ) and also

$$
\frac{v\left(x, s_{n}^{\prime}\right)}{v\left(0, s_{n}^{\prime}\right)}-\cos x \geq 0 \quad \text { on } \quad[-K, K]
$$

for large $n$. Thus by the Arzela-Ascoli theorem we must have $v\left(x, s_{n}^{\prime}\right) / v\left(0, s_{n}^{\prime}\right) \rightarrow$ $\cos x$ (passing to a subsequence if necessary) uniformly on $[-K, K]$ as $n \rightarrow \infty$.

Let $K_{j}$ be a sequence with $K_{j} \rightarrow \pi / 2$ as $j \rightarrow \infty$. For each $j$, there is a sequence $s_{n}^{(j)}$ so that $v\left(x, s_{n}^{(j)}\right) / v\left(0, s_{n}^{(j)}\right) \rightarrow \cos x$ uniformly on $\left[-K_{j}, K_{j}\right]$ as $n \rightarrow \infty$. By a diagonal argument, there is a sequence $\lambda_{n} \nearrow T_{\max }$ such that $v\left(x, \lambda_{n}\right) / v\left(0, \lambda_{n}\right)$ converges uniformly to $\cos x$ on $[-K, K]$ for any $0<K<\pi / 2$.

To obtain the convergence (62) on $[-\pi / 2, \pi / 2]$, we argue as follows (for convenience, any further subsequence of $\lambda_{n}$ is still denoted as $\left.\lambda_{n}\right)$. Assume $v\left(x, \lambda_{n}\right) /$ $v\left(0, \lambda_{n}\right)$ does not converge uniformly to $\cos x$ on $[-\pi / 2, \pi / 2]$. Then there exist $\varepsilon>0$, a sequence of points $x_{n} \in[-\pi / 2, \pi / 2]$, and a time subsequence $\lambda_{n}$ so that

$$
f\left(x_{n}, \lambda_{n}\right):=\frac{v\left(x_{n}, \lambda_{n}\right)}{v\left(0, \lambda_{n}\right)}-\cos x_{n} \geq \varepsilon \text { for all } n .
$$


By the above discussion we may assume that $x_{n} \rightarrow \pi / 2$. Now by the mean value theorem and (16)

$$
\begin{aligned}
\varepsilon & <\frac{v\left(x_{n}, \lambda_{n}\right)}{v\left(0, \lambda_{n}\right)} \leq \frac{\left|v\left(x_{n}, \lambda_{n}\right)-v\left(x_{n}-\varepsilon / 100, \lambda_{n}\right)\right|}{v\left(0, \lambda_{n}\right)}+\frac{v\left(x_{n}-\varepsilon / 100, \lambda_{n}\right)}{v\left(0, \lambda_{n}\right)} \\
& \leq \frac{\varepsilon}{100}+\frac{v\left(x_{n}-\varepsilon / 100, \lambda_{n}\right)}{v\left(0, \lambda_{n}\right)}
\end{aligned}
$$

where $v\left(x_{n}-\varepsilon / 100, \lambda_{n}\right) / v\left(0, \lambda_{n}\right) \rightarrow \cos (\pi / 2-\varepsilon / 100)$ as $n \rightarrow \infty$. We have a contradiction.

Since $v\left(x, \lambda_{n}\right) / v\left(0, \lambda_{n}\right)$ is decreasing in $x \in(0, m \pi)$ for each time $\lambda_{n}$, it must converge to zero uniformly outside the interval $[-\pi / 2, \pi / 2]$. The proof of Theorem 25 is done.

We next want to improve Theorem 25] and show that the convergence in (62) is valid for all $t \rightarrow T_{\max }$, not just along a sequence of times $t_{n} \nearrow T_{\max }$. Below, we basically follow similar arguments as in Lemmas 4.4, 4.5, and 4.6 of FriedmanMcLeod [FM] and look more closely at the solution behavior. These estimates are also interesting on their own.

In the following we still assume that the initial data $v_{0}(x)$ satisfies the symmetric condition (57).

Lemma 27. If $x \in(\pi / 2, \pi)$, then

$$
\frac{d}{d t} \int_{0}^{x} v^{1-p}(y, t) \cos y d y<0 \quad \text { for all } \quad t \in\left[0, T_{\max }\right) .
$$

Proof. We proceed as in [FM], Lemma 4.5. By direct computation, we have

$$
\frac{d}{d t} \int_{0}^{x} v^{1-p}(y, t) \cos y d y=(1-p)\left[v_{x}(x, t) \cos x+v(x, t) \sin x\right]<0,
$$

since for $x \in(\pi / 2, \pi)$ we have $v_{x}(x, t)<0, \cos x<0, \sin x>0$.

Lemma 28. If $x>\pi / 2$ or $x<-\pi / 2$, then there exists a constant $C$ depending on $x$ such that

$$
0<v(x, t) \leq C \quad \text { for all } \quad t \in\left[0, T_{\max }\right)
$$

i.e., $v(x, t)$ does not blow up for $|x|>\pi / 2$.

Proof. We proceed as in $[\overline{F M}$, Lemma 4.6. Since $v(x, t)$ is decreasing in $x \in(0, m \pi)$ for all time, without loss of generality, we may just look at the case $x \in(\pi / 2, \pi)$. Suppose $v(x, t)$ is not bounded. Then there exists a sequence $t_{n} \nearrow T_{\max }$ so that $v\left(x, t_{n}\right) \rightarrow \infty$. By Lemma 6 we must have $v(x, t) \rightarrow \infty$ as $t \rightarrow T_{\max }$. In particular, we have (note that $v(y, t)$ is decreasing for $y>0$ )

$$
\int_{0}^{x} v^{1-p}(y, t) \cos y d y \rightarrow 0 \quad \text { as } \quad t \rightarrow T_{\max } .
$$


On the other hand, we may write for fixed small $\delta>0$

$$
\begin{aligned}
& \int_{0}^{x} v^{1-p}(y, t) \cos y d y \\
& =\int_{0}^{(\pi-\delta) / 2} v^{1-p}(y, t) \cos y d y+\int_{(\pi-\delta) / 2}^{(\pi+\delta) / 2} v^{1-p}(y, t) \cos y d y \\
& \quad+\int_{(\pi+\delta) / 2}^{x} v^{1-p}(y, t) \cos y d y .
\end{aligned}
$$

As $v(x, t)>0$ is decreasing in $x \in(0, m \pi)$, the second term in (78) is negative for all time. Also by (17) in Lemma 7] there is a constant $c>0$ such that $v(y, t) \geq c v(0, t)$ for all $y \in[0,(\pi-\delta) / 2]$ and all time large enough. Finally, for $y \in[(\pi+\delta) / 2, x]$, by Theorem 25 there exists a sequence $t_{n} \nearrow T_{\max }$ such that $v\left(y, t_{n}\right) \leq \varepsilon_{n} v\left(0, t_{n}\right)$, where $\varepsilon_{n} \rightarrow 0$ as $n \rightarrow \infty$. Hence for $n$ large enough we conclude

$$
\begin{aligned}
& \int_{0}^{x} v^{1-p}\left(y, t_{n}\right) \cos y d y \\
& \leq c^{1-p} v^{1-p}\left(0, t_{n}\right) \int_{0}^{(\pi-\delta) / 2} \cos y d y+\varepsilon_{n}^{1-p} v^{1-p}\left(0, t_{n}\right) \int_{(\pi+\delta) / 2}^{x} \cos y d y<0 .
\end{aligned}
$$

This gives a contradiction due to (75) and (77).

The proof for the case $x<-\pi / 2$ is similar.

Lemma 29. Let $w(x)$ be a nonnegative Lipschitz function defined on $[-\pi / 2, \pi / 2]$. Suppose that $w$ satisfies the inequality $w_{x x}+w \geq 0$ on $(-\pi / 2, \pi / 2)$ in the sense of distribution. If $w(-\pi / 2)=w(\pi / 2)=0$, then $w(x)=a \cos x$, where $a=$ $\max _{x \in[-\pi / 2, \pi / 2]} w(x)$.

Proof. Let $\varphi_{n}$ be a sequence of smooth nonnegative functions with compact support in $(-\pi / 2, \pi / 2)$ such that it converges to $w$ in $H_{0}^{1}[-\pi / 2, \pi / 2]$ (note that $0 \leq w \in$ $\left.H_{0}^{1}[-\pi / 2, \pi / 2]\right)$. Since $w$ satisfies $w_{x x}+w \geq 0$ on $(-\pi / 2, \pi / 2)$ in the sense of distribution, we have

$$
\int_{-\frac{\pi}{2}}^{\frac{\pi}{2}}\left(\varphi_{n}^{\prime}(x) w^{\prime}(x)-\varphi_{n}(x) w(x)\right) d x \leq 0 \quad \text { for all } n .
$$

Letting $n \rightarrow \infty$ we get

$$
\int_{-\frac{\pi}{2}}^{\frac{\pi}{2}}\left(\left(w^{\prime}(x)\right)^{2}-w^{2}(x)\right) d x \leq 0
$$

Note that $\lambda=1$ is the principal eigenvalue of the operator $d^{2} / d x^{2}$ on the interval $[-\pi / 2, \pi / 2]$, with $\cos x$ the principal eigenfunction satisfying the Dirichlet boundary condition. Thus we obtain

$$
\int_{-\frac{\pi}{2}}^{\frac{\pi}{2}} w^{2}(x) d x \leq \int_{-\frac{\pi}{2}}^{\frac{\pi}{2}}\left(w^{\prime}(x)\right)^{2} d x
$$

where equality holds only when $w$ is a constant multiple of the principal eigenfunction. Equations (79) and (80) imply that $w$ is a principal eigenfunction on the interval $[-\pi / 2, \pi / 2]$, and so $w_{x x}+w=0$ on $(-\pi / 2, \pi / 2)$. Let $a=\max _{x \in[-\pi / 2, \pi / 2]} w(x)$. Then we conclude that $w=a \cos x$ on $[-\pi / 2, \pi / 2]$. 
Theorem 30. Under the same assumption as in Theorem 25] we have

$$
\lim _{t \rightarrow T_{\max }} \frac{v(x, t)}{v(0, t)}=\Phi(x) \quad \text { uniformly on } \quad x \in[-m \pi, m \pi] .
$$

Proof. It suffices to prove that for any sequence $t_{j} \nearrow T_{\max }$ there is a subsequence, also denoted as $t_{j}$, so that

$$
\lim _{j \rightarrow \infty} \frac{v\left(x, t_{j}\right)}{v\left(0, t_{j}\right)}=\Phi(x) \quad \text { uniformly on } \quad x \in[-m \pi, m \pi] .
$$

This would imply that the convergence is for all time $t \rightarrow T_{\max }$. Let $t_{j}$ be a sequence with $t_{j} \nearrow T_{\max }$. By Lemma 6 there is a subsequence $t_{j}$ and a nonnegative Lipschitz function $w(x)$ defined on $[-m \pi, m \pi]$ so that

$$
\lim _{j \rightarrow \infty} \frac{v\left(x, t_{j}\right)}{v\left(0, t_{j}\right)}=w(x) \quad \text { uniformly on } \quad x \in[-m \pi, m \pi] .
$$

We clearly have $\max _{x \in[-\pi / 2, \pi / 2]} w=1$, and by Lemma [6] it satisfies $w_{x x}+w \geq 0$ on $(-\pi / 2, \pi / 2)$ in the sense of distribution. By Lemma 28 since $v(x, t)$ does not blow up for $|x|>\pi / 2$, we must have $w(x)=0$ for $|x|>\pi / 2$. By continuity, we have $w(-\pi / 2)=w(\pi / 2)=0$. Thus Lemma 29 implies that $w(x)=\cos x$ for $|x|<\pi / 2$. The proof is done.

4.2. Convergence to a translational self-similar solution. Going back to the slow-speed curve contracting flow $(\star)$ with $\alpha \in(0,1]$, the initial curve $\gamma_{0}$ has curvature $k_{0}(x)>0$ satisfying (יㅡ), i.e.,

$$
\int_{S_{m}^{1}} \frac{1}{k_{0}(x)} e^{i x} d x=0
$$

If $k_{0}(x)$ satisfies the symmetric condition

$$
k_{0}(x)=k_{0}(-x) \quad \text { and } \quad k_{0}^{\prime}(x)<0, \quad x \in(0, m \pi),
$$

and $k_{\max }(t)$ of $\gamma_{t}$ has type-two blow-up, then by Theorem 30 we have the convergence

$$
\lim _{t \rightarrow T_{\max }} \frac{k(x, t)}{k(0, t)}=(\cos x)^{\frac{1}{\alpha}} \quad \text { uniformly on } \quad x \in[-\pi / 2, \pi / 2] .
$$

It is well known that under the flow $(\star)$ there is a special translational selfsimilar solution $\Gamma_{t}$ translating in the direction $(0,1)$ with unit speed (see [NT] or the book [CZ]). For each time $t, \Gamma_{t}$ is only a translation of $\Gamma_{0}$ (this $\Gamma_{0}$ is not a closed curve, but still convex and embedded). If we use tangent angle $x$ to parametrize $\Gamma_{0}$, its parametrization is given by

$$
\Gamma_{0}=\left(\int_{0}^{x} \frac{\cos \xi}{(\cos \xi)^{\frac{1}{\alpha}}} d \xi, \int_{0}^{x} \frac{\sin \xi}{(\cos \xi)^{\frac{1}{\alpha}}} d \xi\right), \quad x \in(-\pi / 2, \pi / 2),
$$

where

$$
\int_{0}^{x} \frac{\sin \xi}{(\cos \xi)^{\frac{1}{\alpha}}} d \xi=\left\{\begin{array}{l}
\frac{\alpha}{\alpha-1}\left[1-(\cos x)^{1-\frac{1}{\alpha}}\right], \quad \alpha \in(0,1), \\
-\log \cos x, \quad \alpha=1 .
\end{array}\right.
$$


In particular, the curve $\Gamma_{0}$ goes to infinity as $x \rightarrow \pm \pi / 2$. The curvature of $\Gamma_{0}$ at angle $x$ is given by $k(x)=(\cos x)^{1 / \alpha}, x \in(-\pi / 2, \pi / 2)$, with maximum at $x=0$. When $\alpha=1$, we get Grayson's "Grim Reaper", which is $\Gamma_{0}=(x,-\log \cos x), x \in$ $(-\pi / 2, \pi / 2)$.

Evolve the above given symmetric $\gamma_{0}$ according to the flow $(\star)$. For any $t \in\left[0, T_{\max }\right)$, choose the point $x_{t} \in \gamma_{t}$ at which the curvature is $k_{\max }(t)$ (by the assumption there is only one such point) and translate $\gamma_{t}$ so that $x_{t}$ becomes the origin $O=(0,0)$. Call this translational curve $\tilde{\gamma}_{t}$. Next rotate it so that the unit tangent vector at the origin of $\tilde{\gamma}_{t}$ becomes $(1,0)$, and finally dilate the curve so that its maximal curvature becomes 1 and denote this final curve as $\hat{\gamma}_{t}$. Theorem 30 says that if we have type-two blow-up of $k_{\max }(t)$, then over the region $x \in(-\pi / 2, \pi / 2)$, $\hat{\gamma}_{t}$ converges to the above translational self-similar solution $\Gamma_{0}$ as $t \rightarrow T_{\max }$. When $\alpha=1$, this phenomenon has been observed by Angenent in [ANG.

Thus we can summarize the following important observation of the slow speed flow $(\star)$ : for type-one blow-up, the asymptotic behavior is given by a homothetic self-similar solution, while for type-two blow-up, the asymptotic behavior (in the special symmetric case) is given by a translational self-similar solution.

To end this paper we point out that most of the lemmas and theorems remain valid. Even the initial condition $v_{0}(x)$ does not satisfy the integral condition (3), as long as it is positive, smooth, and $2 m \pi$-periodic. They include Lemmas 5, 6, 7 , 27 and Theorems 8, 10, 18.

As for Lemma 28 and Theorems 25, 30, if we add the extra assumption that $\left(T_{\max }-t\right)^{1 / p} v_{\max }(t)$ is not bounded on $t \in\left[0, T_{\max }\right)$, then they are all valid even if $v_{0}(x)$ does not satisfy (3).

In particular, we emphasize again that for $p \in[2, \infty)$, there is either type-one blow-up or type-two blow-up. Moreover, type-one blow-up occurs only when $v_{0}(x)$ satisfies the integral condition (3). If $v_{0}(x)$ does not satisfy (3), then the blow-up is always of type-two. Thus the generic blow-up behavior for $p \in[2, \infty)$ is type-two.

4.3. What to do next? There is still a difficult question of estimating the typetwo blow-up rate of $v(x, t)=k^{\alpha}(x, t)$. When $\alpha=1$ (i.e., $p=1+1 / \alpha=2$ ) and $m=2$, Angenent and Velázquez [AV] had given a nontrivial proof of the existence of some symmetric initial data $v_{0}(x)>0$, satisfying (3), with the type-two blow-up rate

$$
v_{\max }(t)=(1+o(1)) \sqrt{\frac{\ln \ln \left(\frac{1}{T_{\max }-t}\right)}{T_{\max }-t}} \text { as } t \rightarrow T_{\max },
$$

and therefore

$$
v_{\max }(t) \sqrt{T_{\max }-t} \sim \sqrt{\ln \ln \left(\frac{1}{T_{\max }-t}\right)} \rightarrow \infty \text { as } t \rightarrow T_{\max }
$$

We are wondering if a certain similar estimate holds in the case $\alpha \in(0,1]$ (i.e., $p>2$ ). At this moment we do not know, and we hope to work on it in the future. 


\section{Some PICTURES FOR THE ODE (50)}

In this section we give some pictures relating to the $\mathrm{ODE} w^{\prime \prime}+w-w^{1-p}=$ 0 . These pictures can help us understand convergence behavior (for general $p \in$ $(-\infty, \infty)$ ) of the PDE $\partial u / \partial \tau=u^{p}\left(u_{x x}+u-u^{1-p}\right)$ (with positive initial data $u_{0} \in C^{\infty}\left(S_{m}^{1}\right)$ and periodic boundary condition). This is because the ODE is a steady state of the PDE. Let

$$
F(s)=\left\{\begin{array}{l}
s^{2}-\frac{2}{2-p} s^{2-p}, \quad p \neq 2, \quad p \in(-\infty, \infty), \quad s \in(0, \infty) . \\
s^{2}-2 \log s, \quad p=2,
\end{array}\right.
$$

The graphs of $F(s)$ for $p=-1 \in(-\infty, 0), p=1 \in(0,2), p=3 \in[2, \infty)$ are given below:
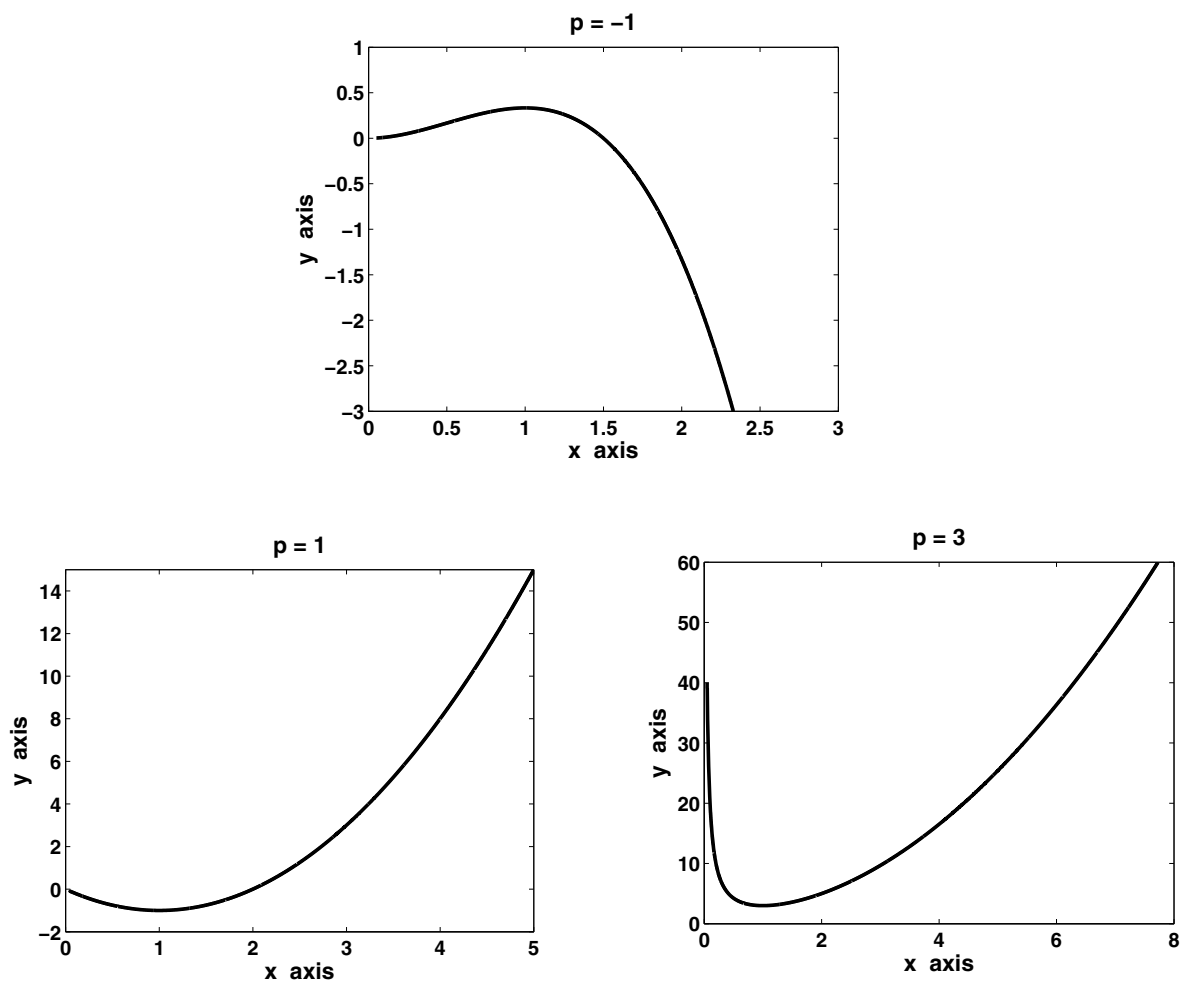

The graph of $F(s)$ for any $p \in(-\infty, 0)$ is analogous to the above picture for $p=-1 . F(s)$ increases on $s \in(0,1)$ with $F(0)=0, F(1)>0$, and decreases to $-\infty$ on $(1, \infty)$. The graph of $F(s)$ for any $p \in(0,2)$ is analogous to the above picture for $p=1$. $F(s)$ decreases on $s \in(0,1)$ with $F(0)=0, F(1)<0$, and increases to $+\infty$ on $(1, \infty)$. Finally, the graph of $F(s)$ for any $p \in[2, \infty)$ is analogous to the above picture for $p=3$. $F(s)$ decreases on $s \in(0,1)$ with $\lim _{s \rightarrow 0^{+}} F(s)=+\infty$, $F(1)>0$, and increases to $+\infty$ on $(1, \infty)$. Also note that when $p=0, F(s) \equiv 0$.

Any solution $w(x)$ to the ODE $w^{\prime \prime}+w-w^{1-p}=0$ must satisfy the identity $w_{x}^{2}(x)=F(M)-F(w(x))$ for all $x$ in the domain $i$ of $w(x)$, on which $w(x)>0$. Here we may assume $0 \in I$, and $w(0)=M \geq 1$ is the maximum value of $w$ on $I$. For $p \in(-\infty, 0)$, we only have type-one blow-up for $v(x, t)$ of equation (\$). If the rescaled solution $u(x, t)=v(x, t) / R(t)$ converges to $w(x)$ on some interval $I$, 
then we must have $w(x) \equiv 1$ over $I$. Otherwise we have $M>1$ and use the first picture to get

$$
w_{x}^{2}(x)=F(M)-F(w(x))<0
$$

for all $x \in I$ such that $1 \leq w(x)<M$. This gives a contradiction, and so $w(x) \equiv 1$ over $I$.

The main difference between $p \in(0,2)$ and $p \in[2, \infty)$ is that there exist bump solutions (degenerate) to the ODE for $p \in(0,2)$, but for $p \in[2, \infty)$ all solutions to the ODE are positive everywhere and periodic over $\mathbb{R}$ (nondegenerate). Again, this can also be seen from the second and third pictures.

\section{ACKNOWLEDGMEnTs}

The authors owe a great deal to the referee of this paper, whose comments, suggestions, and corrections have been very helpful. While writing this paper, the authors had discussions with Professors Ben Andrews, Sigurd Angenent and Hiroshi Matano. They thank all of them. Finally, the third author would like to acknowledge the support of the National Science Council and the National Center for Theoretical Sciences of Taiwan.

\section{REFERENCES}

[AL] Abresch, U., Langer, J. (1986) The normalized curve shortening flow and homothetic solutions, J. Diff. Geom., 23, 175-196. MR845704 (88d:53001)

[AN1] Andrews, B. (1998) Evolving convex curves, Cal. of Var. \& PDE., 7, no. 4, 315-371. MR $1660843(99 \mathrm{k}: 58038)$

[AN2] Andrews, B. (1994) Harnack inequality for evolving hypersurfaces, Math. Zeit., 217, 179-197. MR:1296393 (95j:58178)

[AN3] Andrews, B. (2003) Classification of limiting shapes for isotropic curve flows, J. of the AMS, 16, no. 2, 443-459. MR1949167 (2004a:53083)

[AN4] Andrews, B. (2002) Non-convergence and instability in the asymptotic behavior of curves evolving by curvature, Comm. Anal. \& Geom., vol. 10, no. 2, 409-449. MR1900758 (2003e:53086)

[ANG] Angenent, S. (1991) On the formation of singularities in the curve shortening flow, J. Diff. Geom., 33, 601-633. MR.1100205 (92c:58016)

[AV] Angenent, S., Velázquez, J. J. L. (1995) Asymptotic shape of cusp singularities in curve shortening, Duke Math. J., vol. 77, no. 1, 71-110. MR.1317628 (95k:58036)

[C] Chow, B. (1997) Geometric aspects of Aleksandrov reflection and gradient estimates for parabolic equations, Comm. Anal. Geom., vol. 5, no. 2, 389-409. MR1483984 (98k:53045)

[CLT] Chow, B., Liou, L. P., Tsai, D. H. (1996) Expansion of embedded curves with turning angle greater than- $\pi$, Invent. Math., 123, 415-429. MR.1383955 (97c:58025)

$[\mathrm{CM}]$ Chen, X. Y., Matano, H. (1989) Convergence, asymptotic periodicity, and finite-point blow-up in one-dimensional semilinear heat equations, J. Diff. Eq., 78, 160-190. MR.986159 (90e:35018)

[CPE] Cortazar, C., Del Pino, M., Elgueta, M. (1998) On the blow-up set for $\partial_{t} u=\triangle u^{m}+$ $u^{m}, m>1$, Indiana Univ. Math. J., 47, p. 541-561. MR.1647932 (99h:35085)

[CT] Chow, B., Tsai, D. H. (1996) Geometric expansion of convex plane curves, J. Diff. Geom., 44, 312-330. MR1425578 (97m:58041)

[CZ] Chou, K. S., Zhu, X. P. (2000) The Curve Shortening Problem, Chapman and Hall/ CRC. MR1888641 (2003e:53088)

[FS] Feireisl, E. and Simondon, F. (1999) Convergence for degenerate parabolic equations, J. Diff. Eq., vol. 152, 439-466. MR.1674569 (2000d:35123)

[FM] Friedman, A., McLeod (1986) Blow-up of solutions of nonlinear degenerate parabolic equations, Arch. Rational Mech. Anal., 96, 55-80. MR853975 (87j:35051) 
[GA] Gage, M. (1984) Curve shortening makes convex curves circular, Invent. Math., 76, 357-364. MR742856 (85i:52004)

[GH] Gage, M., Hamilton, R. S. (1986) The heat equation shrinking convex plane curves, J. Diff. Geom., 23, 69-96. MR840401 (87m:53003)

[GR] Grayson, M. (1987) The heat equation shrinks embedded plane curves to round points, J. Diff. Geom., 26, 285-314. MR906392 (89b:53005)

[LPT] Lin, T. C., Poon, C. C., Tsai, D. H. (2009) Expanding convex immersed closed plane curves, Cal. of Var. \& PDEs., 34, 153-178. MR2448648 (2010m:35191)

[LT1] Lin, Y. C., Tsai, D. H. (2008) On a simple maximum principle technique applied to equations on the circle, J. Diff. Eqs., 245, 377-391. MR2428003(2010b:35252)

[LT2] Lin, Y. C., Tsai, D. H. (2009) Evolving a convex closed curve to another one via a lengthpreserving linear flow, J. Diff. Eqs., 247, 2620-2636. MR2568066 (2010j:53135)

[NT] Nien, C. H., Tsai, D. H. (2006) Convex curves moving translationally in the plane, J. of Diff. Eq., 225, 605-623. MR2225802 (2007a:35070)

[PT] Poon, C. C., Tsai, D. H. (2010) Contracting convex immersed closed plane curves with fast speed of curvature, Comm. Anal. \& Geom., vol. 18, no. 1, 23-75. MR2660457 (2011g:53146)

[T1] Tsai, D. H. (2000) Blowup and convergence of expanding immersed convex plane curves, Comm. Anal. \& Geom., vol. 8, no. 4, 761-794. MR1792373 (2001k:53133)

[T2] Tsai, D. H. (2005) Behavior of the gradient for solutions of parabolic equations on the circle, Cal. of Var. \& PDE., vol. 23, 251-270. MR2142063 (2006d:35116)

[T3] Tsai, D. H. (2004) Blowup behavior of an equation arising from plane curves expansion, Diff. and Integ. Eq., vol. 17, no. 7-8, 849-872. MR2075410(2005e:35120)

[T4] Tsai, D. H. (2005) Asymptotic closeness to limiting shapes for expanding embedded plane curves, Invent. Math., 162, 473-492. MR.2198219(2006j:53099)

[TSO] K. Tso, (1985) Deforming a hypersurface by its Gauss-Kronecker curvature, Comm. Pure and Appl. Math., 38, 867-882. MR812353(87e:53009)

[U1] Urbas, J. (1991) An expansion of convex hypersurfaces, J. Diff. Geom., 33, 91-125. MR.1085136 (91j:58155)

[U2] Urbas, J. (1999) Convex curves moving homothetically by negative powers of their curvature, Asian J. Math., vol. 3, no. 3, 635-658. MR.1793674 (2001m:53119)

Department of Mathematics, National Cheng Kung University, Tainan 701, Taiwan

E-mail address: yclin@math.ncku.edu.tw

Department of Mathematics, National Chung Cheng University, Chiayi 621, Taiwan

E-mail address: ccpoon@math.ccu.edu.tw

Department of Mathematics, National Tsing Hua University, Hsinchu 300, Taiwan

E-mail address: dhtsai@math.nthu.edu.tw 